\title{
Impairment of Pattern Separation of Ambiguous Scenes by Single Units in the CA3 in the Absence of the Dentate Gyrus
}

\author{
Choong-Hee Lee and Inah Lee \\ Department of Brain and Cognitive Sciences, Seoul National University, Seoul 08826, South Korea
}

Theoretical models and experimental evidence have suggested that connections from the dentate gyrus (DG) to CA3 play important roles in representing orthogonal information (i.e., pattern separation) in the hippocampus. However, the effects of eliminating the DG on neural firing patterns in the CA3 have rarely been tested in a goal-directed memory task that requires both the DG and CA3. In this study, selective lesions in the DG were made using colchicine in male Long-Evans rats, and single units from the CA3 were recorded as the rats performed visual scene memory tasks. The original scenes used in training were altered during testing by blurring to varying degrees or by using visual masks, resulting in maximal recruitment of the DG-CA3 circuits. Compared with controls, the performance of rats with DG lesions was particularly impaired when blurred scenes were used in the task. In addition, the firing rate modulation associated with visual scenes in these rats was significantly reduced in the single units recorded from the CA3 when ambiguous scenes were presented, largely because DGdeprived CA3 cells did not show stepwise, categorical rate changes across varying degrees of scene ambiguity compared with controls. These findings suggest that the DG plays key roles not only during the acquisition of scene memories but also during retrieval when modified visual scenes are processed in conjunction with the CA3 by making the CA3 network respond orthogonally to ambiguous scenes.

Key words: CA3; dentate gyrus; hippocampus; pattern completion; pattern separation; place cell

Significance Statement

Despite the behavioral evidence supporting the role of the dentate gyrus in pattern separation in the hippocampus, the underlying neural mechanisms are largely unknown. By recording single units from the CA3 in DG-lesioned rats performing a visual scene memory task, we report that the scene-related modulation of neural firing was significantly reduced in the DGlesion rats compared with controls, especially when the original scene stimuli were ambiguously altered. Our findings suggest that the dentate gyrus plays an essential role during memory retrieval and performs a critical computation to make categorical rate modulation occur in the CA3 between different scenes, especially when ambiguity is present in the environment.

\section{Introduction}

The hippocampus plays an essential role in remembering events as discrete episodic memories (Eichenbaum, 2000). Computational models have suggested that forming and retrieving distinct memory representations in the hippocampus require the following two key processes: pattern separation and pattern completion (O'Reilly and McClelland, 1994; Treves and Rolls, 1994). Pattern separation consists of the formation of orthogonal representations despite similar input patterns, whereas pattern completion consists of the retrieval

\footnotetext{
Received Oct. 31, 2019; revised Mar. 5, 2020; accepted Mar. 10, 2020.

Author contributions: C.-H.L. and I.L. designed research; C.-H.L. performed research; C.-H.L. and I.L. analyzed data; C.-H.L. and I.L. wrote the paper.

The authors declare no competing financial interests.

This study was supported by the BK21 + program (Grant 5286-2014100), the Basic Research Laboratory Program (Grant 2018R1A4A1025616), and the Brain Research Program (Grants 2019R1A2C2088799, 2017M3C7A1029661) through the National Research Foundation of Korea.

Correspondence should be addressed to Inah Lee at inahlee@snu.ac.kr.

https://doi.org/10.1523/JNEUROSCI.2596-19.2020

Copyright $\odot 2020$ the authors
}

of original memory representations when presented with modified or ambiguous stimuli. Computational models have suggested that different subregions of the hippocampus are responsible for pattern separation and completion processes (O'Reilly and McClelland, 1994; Treves and Rolls, 1994; Kesner and Rolls, 2015). Specifically, some computational models have emphasized that the dentate gyrus (DG) subregion in the hippocampus is critical to the formation of orthogonal representations of events and places in the CA3, the hippocampal structure downstream of the DG. According to these models, the CA3 is important in recovering the original memory representation (i.e., pattern completion) when slightly modified inputs are fed from the cortical areas, mainly due to the recurrent network component in the CA3. It has been hypothesized that two seemingly opposite computational processes performed in the hippocampus are critical in classifying a current environment based on prior original experience.

Experimental evidence using rodents has provided support for these computational models (McNaughton et al., 1989; Gilbert et al., 1998, 2001; Lee and Kesner, 2004; Leutgeb et al., 
2004, 2007; Lee et al., 2004a,b, 2015; Gilbert and Kesner, 2006; Lee and Solivan, 2010; Neunuebel and Knierim, 2014; van Dijk and Fenton, 2018). For example, behavioral studies using rats with lesions in the DG found significant impairment in memory retrieval when performing tasks requiring the differentiation of similar locations (Gilbert et al., 2001) or similar object-place paired associates (Lee and Solivan, 2010). We recently reported that DG lesions impair the acquisition of new visual scenes and that rats with DG lesions were deficient in recognizing visual scenes when the original scenes were altered to make them ambiguous (Ahn and Lee, 2014). These findings indicate that DG is important for disambiguating similar experiences. Studies using human subjects have also reported the roles of the hippocampus in pattern separation (Kirwan and Stark, 2007; Bakker et al., 2008; Lacy et al., 2011; Motley and Kirwan, 2012; Brown et al., 2014; Berron et al., 2016). However, these studies provide limited information since the distinction between the DG and CA3 is difficult to make using the current fMRI techniques. These technical challenges also apply to the electrophysiological characterization of the DG/CA3 areas in pattern separation in nonhuman primates (Sakon and Suzuki, 2019).

Physiologically, very few granule cells are active during behavior in the DG, and those active ones tend to fire only in a single environment (Danielson et al., 2017; GoodSmith et al., 2017; Senzai and Buzsáki, 2017), suggesting their roles in pattern separation (Nakazawa, 2017). In contrast, electrophysiological studies of the CA3 have yielded evidence for both pattern separation (Leutgeb et al., 2007) and pattern completion (Lee et al., 2004b; Neunuebel and Knierim, 2014). Heterogeneity may be present along the transverse axis of the CA3, with the proximal and distal CA3 specialized for pattern separation and pattern completion, respectively (Lee et al., 2015). Most previous physiological studies, however, were conducted using behavioral paradigms that did not require rats to use such computational processes to guide their behavior. Although physiological recordings were made after the DG became dysfunctional in mnemonic tasks in some studies, there was a lack of control over stimulus ambiguity (McNaughton et al., 1989; Sasaki et al., 2018; van Dijk and Fenton, 2018). More importantly, these studies did not characterize the changes in neural activity in the CA3 that resulted from the absence of the DG network.

In the current study, selective lesions were generated in the DG using colchicine, and single units were recorded from the CA3 in animals that had been subjected to a visual scene-based memory task involving altered, ambiguous scenes. Compared with controls, rats lacking the DG showed significant impairment of the rate-modulating capability of single units in the CA3 when required to recognize ambiguous visual scenes.

\section{Materials and Methods}

\section{Subjects}

Eight male rats (Long-Evans) weighing 300-400 g were used. Food was restricted to maintain the body weight at $85 \%$ of free-feeding weight, and water was available ad libitum. All animals were individually housed with a $12 \mathrm{~h}$ light/dark cycle. All animal protocols were approved by the Institutional Animal Care and Use Committee of the Seoul National University.

\section{Behavioral apparatus}

The behavioral task was conducted on an elevated, T-shaped linear track $(73 \times 8 \mathrm{~cm}$ stem with two $38 \times 8 \mathrm{~cm}$ arms), containing a food well $(2.5 \mathrm{~cm}$ in diameter, $0.8 \mathrm{~cm}$ deep) at the end of each arm (Fig. $1 A)$. Each food well was covered by a black, acrylic washer with a silver reflective sticker attached on the bottom surface to record the moment the rat displaced the washer to access the reward with an optic sensor (Autonics) attached to the center of the food well. One of the food wells was baited during the intertrial interval while the rat was confined in a start box. To prevent the rat from locating the baited food well by possible unintended auditory cues from manipulating the washer on the corresponding side, both washers were adjusted equally during the procedure. A guillotine door-operated start box $(22.5 \times 16 \times 31.5 \mathrm{~cm})$ was attached to the side opposite to the stem. Three LCD monitors displaying visual scene stimuli were installed to surround the arm portions of the T-shaped track. Four optic fiber sensors were inserted along the track at distances from the start-box door of $1,27,47$, and $67 \mathrm{~cm}$ to detect the position of the animal and to control the onset and offset of the scenes. Sensor signals were sent to a data-acquisition system (Digital Lynx SX, Neuralynx) as transistor-transistor logic (TTL) signals. Custom-written software created in MATLAB (MathWorks) and Psychtoolbox was used to control scene stimuli and transmit TTL signals containing trial information to the data-acquisition system. During the task, the experimental room was dimly lit with an array of dim LEDs. A camera was attached to the ceiling to record the position and head direction of the rat at a sampling rate of $30 \mathrm{~Hz}$. The apparatus was surrounded with a black curtain, and white noise was played by two speakers $(80 \mathrm{~dB})$ during behavioral sessions to mask unwanted environmental noises.

\section{Visual scene memory task}

A visual scene memory (VSM) task previously used to show that rats with DG lesions were impaired in the acquisition of visual scene memory and in the retrieval of ambiguous scenes (Ahn and Lee, 2014) was used in the present study. A trial was started by opening the door of the start box. This opening was detected by the optical sensor, which immediately triggered the presentation of the visual scenes on the monitors. The rat ran along the track to the end of the stem and had to choose either the left or right arm, depending on the visual scene, to obtain the food reward (Fig. 1A). A food well was placed at the end of each arm and was covered with a black acrylic disk to prevent the animal from using odor to sense a reward. A quarter piece of cereal (Froot Loops, Kellogg's) was hidden in the food well in the arm that was correctly associated with the scene, and the rat retrieved the hidden reward by pushing aside the black acrylic disk. If the rat chose incorrectly, no reward was provided, and the rat was gently guided back to the start box immediately after making the incorrect choice. The intertrial intervals (ITIs) following correct and incorrect choices were set at 10 and $20 \mathrm{~s}$, respectively.

During each ITI, a reward for the next trial was placed in one of the food wells while the rat was confined to the start box. Four grayscale visual patterns (zebra, pebbles, bamboo, mountains) were used as scene stimuli. The luminance of the visual scenes was equalized to an average intensity of 103 in Adobe Photoshop. Zebra and bamboo scenes were associated with the left food well, whereas pebbles and mountain scenes were associated with the right food well (Fig. 1B). Rats were trained to criterion ( $70 \%$ correct choice for each scene on 2 consecutive days), initially with a pair of visual scenes and subsequently with a second pair of scenes. Training sessions before surgery consisted of 40 trials. The presentation sequence for scenes in a given session was pseudorandomized so that (1) each scene was presented an equal number of times in every 20 trials, and (2) the same food well was not used for reward during four consecutive trials. Surgery was performed once the rats had learned both pairs of scene stimuli to criterion.

\section{Surgery}

Rats trained to criterion were randomly assigned to the lesion group or the control group. Rats were placed under deep anesthesia by intraperitoneal injection of sodium pentobarbital (Nembutal; $65 \mathrm{mg} / \mathrm{kg}$ ), and their heads were fixed in a stereotaxic frame (Kopf Instruments). Anesthesia was maintained with $0.5-2 \%$ isoflurane mixed with $100 \% \mathrm{O}_{2}$ throughout surgery. An incision was made along the midline of the scalp to expose the skull. The stereotaxic adapters were adjusted to place the bregma and lambda on the same horizontal plane. Four small burr holes and one big burr hole were drilled. The small burr holes were used for injection, and the large holes for hyperdrive implantation. Either 
A

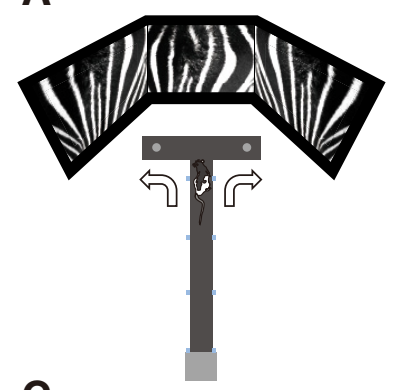

B

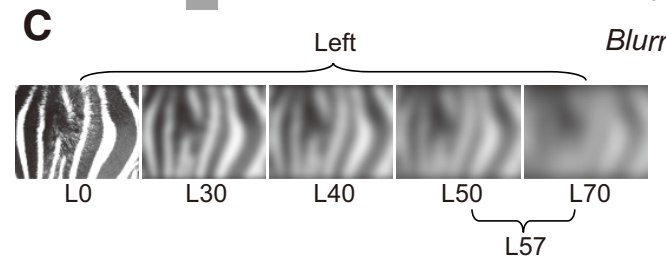

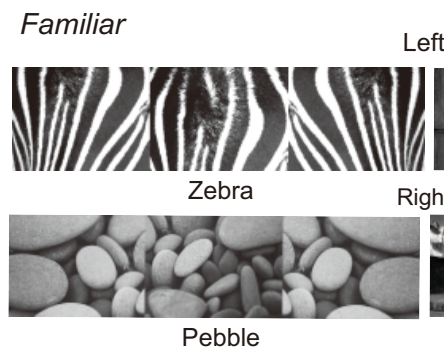

Blurred
Left

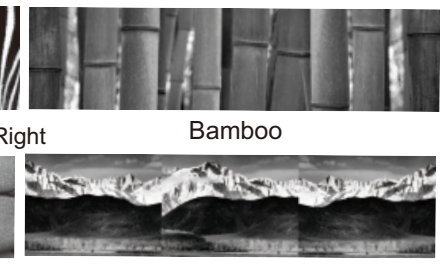

Mountain

Right
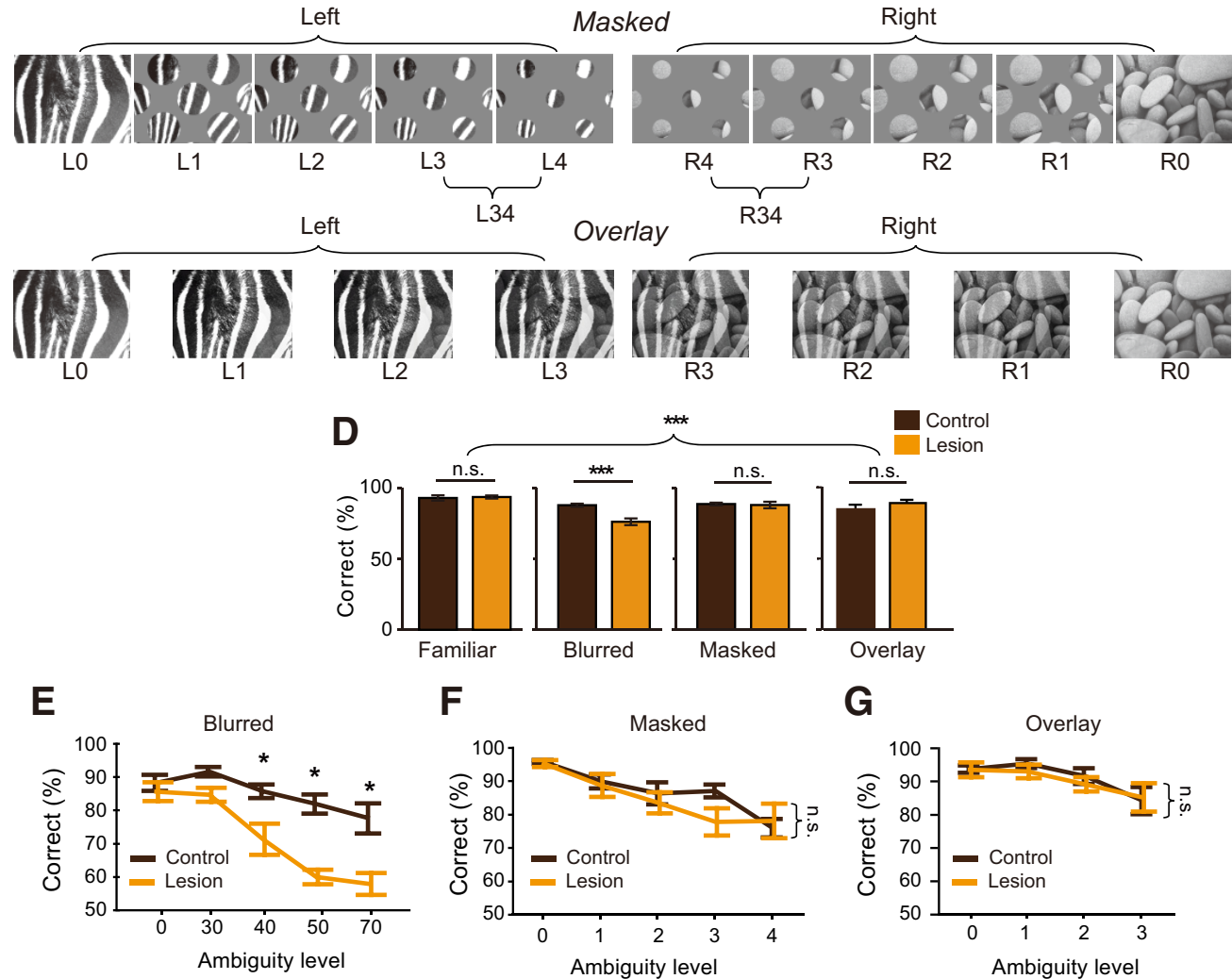

Figure 1. Behavioral paradigm and performance. A, VSM task. B, Four unaltered familiar visual scene stimuli used in Familiar session. C, Top, Blurred session, using 10 Gaussian blurred scenes. Middle, Masked session, using 10 partially occluded scenes. Bottom, Overlay session, using eight partially mixed scenes. In Blurred and Masked sessions, the higher ambiguity levels were grouped together $(L 57, R 57, L 34$, and R34) for the electrophysiological analysis to account for a smaller number of correct trials. $\boldsymbol{D}$, Behavioral performance of the two groups under different scene conditions. Values are given in mean \pm standard error (SE). n.s. not significant, ${ }^{*} p<0.05,{ }^{* *} p<0.01,{ }^{* * *} p<0.001$. $E$, Behavioral performance of the two groups across different ambiguity levels in Blurred sessions. Values are given in mean $\pm \boldsymbol{S E}$. $\boldsymbol{F}$, Same as $\boldsymbol{E}$, but in Masked sessions. Values are given in mean \pm SE. $\boldsymbol{G}$, Same as $\boldsymbol{E}$, but in Overlay sessions. Values are given in mean \pm SE.

colchicine $(7 \mathrm{mg} / \mathrm{ml}, 0.2 \mu \mathrm{l} / \mathrm{site}$ at $10 \mathrm{ml} / \mathrm{h})$ or saline $(0.2 \mu \mathrm{l} /$ site at $10 \mathrm{ml} /$ h) was injected into the dorsal DG at the following coordinates: (1) 2.8 $\mathrm{mm}$ posterior to the bregma, $\pm 1.1 \mathrm{~mm}$ lateral to the midline, and 3.7 $\mathrm{mm}$ ventral from the dura; and (2) $4.0 \mathrm{~mm}$ posterior to the bregma, $\pm 2.3 \mathrm{~mm}$ lateral to the midline, and $3.2 \mathrm{~mm}$ ventral from the dura.

Colchicine is a neurotoxin that, when injected into the hippocampus, selectively ablates granule cells in the DG, while having a minimal effect on the principal cell layers in the CA1 and CA3 (Walsh et al., 1986; Xavier et al., 1999). Colchicine or saline was injected using a custommade $10 \mu$ l glass pipette, with tips at least $8 \mathrm{~mm}$ long to minimize damage to the overlying cortical regions. At each site, the glass pipette was maintained at the injection depth for $2 \mathrm{~min}$ after infusion to avoid the possible upward spread of colchicine. Control animals underwent the same surgical procedures except that artificial CSF (aCSF) was injected into the DG. All injections were made by connecting a 10-ml Hamilton syringe operated with a microinjection pump (Cole-Parmer).

Once injections were finished, a hyperdrive carrying 24 tetrodes and 3 reference electrodes was implanted into each rat to record spikes from single units in the dorsal CA3. The impedance of each tetrode was adjusted to $100-300 \mathrm{k} \Omega$ (measured in gold solution at $1 \mathrm{kHz}$ with an impedance tester) $2 \mathrm{~d}$ before surgery. The surgical coordinates for making the large burr hole was predetermined to allow the tetrodes to cover a range of 2.5-5 mm posterior to the bregma and 2.5-5 mm lateral to the midline. The hyperdrive was affixed to the skull by applying bone 
cement to its bundle and to multiple skull screws around the bundle. After surgery, rats were orally administered diluted ibuprofen syrup to attenuate pain and cared for in a veterinary intensive care unit for $24 \mathrm{~h}$. The details of these surgical procedures have been described previously (Ahn and Lee, 2014; Delcasso et al., 2014).

\section{Electrophysiological recording}

After a $5 \mathrm{~d}$ recovery period, rats were retrained $(\sim 160$ trials per session using the same pairs of scenes used before surgery) until they showed stable performance ( $>75 \%$ correct choices for each scene), during which tetrodes were gradually lowered into target areas. To adjust the tetrodes, the rats were placed on a pedestal in a custom-made aluminum booth outside the behavioral testing room. Neural signals were transmitted through the headstage (HS-36, Neuralynx) and the tether attached to the electrode interface board (Neuralynx) of the hyperdrive to the data-acquisition system. Neural signals were digitized at $32 \mathrm{kHz}$ (filtered at $600-$ $6000 \mathrm{~Hz}$ ) and amplified 1000-10000 times. Tetrodes were lowered daily by small increments to reach the target areas.

Once the electrophysiological recording session began, all four scenes shown during the training period were presented in a pseudorandomized order in a testing session of familiar scenes (Familiar, $\sim 160$ trials per session; Fig. $1 B$ ). During the behavioral task, neural signals were relayed through a slip-ring commutator (Neuralynx) to the data-acquisition system, and an array of green and red LEDs was attached to the headstage to monitor the position and head direction of the animal using a digital camera on the ceiling (sampling rate, $30 \mathrm{~Hz}$ ). Once rats performed well in familiar sessions for 2 consecutive days, they were subjected to a blurred version of the task (Blurred). Each Blurred session began with 10 trials of an unaltered pair of scenes (zebra and pebble scenes on the first day, and bamboo and mountain scenes on the second day), followed by presentation of modified versions of the original pair of scenes (30\%, $40 \%, 50 \%$, and $70 \%$ Gaussian blurred images) along with the original scenes $(\sim 160$ trials per session; Fig. $1 C)$. After $2 \mathrm{~d}$ of Blurred sessions, rats were subjected to Overlay sessions for $2 \mathrm{~d}$, followed by a Masked session. Procedures for the Overlay and Masked sessions were identical to those of the Blurred sessions, except that visual scene stimuli were altered in a different manner. For the Overlay sessions, two scene stimuli with different opacity values were mixed together to create a single visual scene (Fig. 1C, bottom). In the Masked sessions, Gaussian blurred images were replaced by partially occluded images with visual masks composed of viewing holes of four different diameters (Fig. 1C, middle). This manipulation was intended to create partial cues that may require pattern completion. Once the Masked sessions were completed, rats were subjected to a session with novel scenes for $2 \mathrm{~d}$. The results of the Novel scene sessions are not included in the current study.

\section{Histology}

Once all recording sessions were concluded, the rats were subjected to histological procedures to assess the extent of the neurotoxic lesions and to determine the locations of the tetrodes. Rats inhaled overdose of $\mathrm{CO}_{2}$ and transcardially perfused with a solution of $4 \%(\mathrm{v} / \mathrm{v})$ formaldehyde in $0.1 \mathrm{M}$ PBS. The brain was extracted and stored in a $30 \%$ sucrose-formalin solution until it sank. The brain was gelatin-coated, again soaked in $30 \%$ sucrose-formalin solution, and sectioned at $40 \mu \mathrm{m}$ depth using a freezing microtome (model HM 430, Thermo Fisher Scientific). The brain sections were mounted and stained with thionin, and photomicrographs of these sections were taken with a digital camera (Eclipse 80i, Nikon) attached to a microscope at $1 \times$ magnification. Brains were reconstructed from these sections to match the configuration of the tetrodes of the presurgical bundle design. The exact locations of tetrodes were determined using the 3D reconstructed images and physiological depth profiles recorded during the tetrode adjustment period. To examine the integrity of cell layers in the DG and CA3, additional photomicrographs of the brain sections were taken with a digital slide scanner (MoticEasyScan, Motic) at $40 \times$ magnification.

\section{$3 \mathrm{D}$ volumetry}

To evaluate the effect of colchicine infusion, we conducted a $3 \mathrm{D}$ volumetric analysis (Lee et al., 1999; Ahn and Lee, 2014) for the following
Table 1. The number of CA3 units included in the analysis after unit sorting and place-field definition

\begin{tabular}{llll}
\hline Session & Group & Complex spiking units & Units with intact place field \\
\hline Familiar & Control & 58 & 35 \\
& DG lesion & 84 & 45 \\
& Total & 142 & 80 \\
Blurred & Control & 47 & 24 \\
& DG lesion & 43 & 16 \\
& Total & 90 & 40 \\
Masked & Control & 84 & 51 \\
& DG lesion & 61 & 29 \\
\multirow{4}{*}{ Overlay } & Total & 145 & 80 \\
& Control & 71 & 32 \\
& DG lesion & 76 & 27 \\
& Total & 147 & 59 \\
\hline
\end{tabular}

The number of CA3 units from each session per group are shown. Complex spiking units were used for comparing basic firing properties, and the units with intact place fields were used for rate-modulation analysis.

hippocampal subregions: CA1, CA3, and DG. Among the thionin-stained brain sections acquired, 18 brain sections from 2 to $4 \mathrm{~mm}$ posterior to bregma were used for volumetric analysis. In each section, principal cell layers were traced with a color corresponding to one of the hippocampal subregions. For each subregion, we calculated the percentage voxel index of individual rats, in which the mean of the number of colored pixels from four control rats was set as $100 \%$ (ImageJ, National Institutes of Health). For statistical comparison, we performed a two-way ANOVA with group and subregion as factors, implemented in JMP 11 statistical software package (SAS Institute). The results of post-hoc $t$ tests comparing groups and subregions were corrected using a Bonferroni correction procedure for multiple-comparison corrections. To ensure that the recording locations between the control and DG-lesion groups were homogeneous, we calculated the relative position of each tetrode by measuring the length of the CA3 layer; the relative position of 0 denotes the most proximal CA3, whereas 1 denotes the boundary between CA3 and CA2 pyramidal layers.

\section{Unit isolation}

Single units from the dorsal CA3 were isolated manually using customwritten software (WinClust). Various waveform parameters such as peak and energy were used during the procedure as described previously (Delcasso et al., 2014). Neurons were included in analyses if they showed $\geq 50$ spikes during an outbound journey from the start box to the food well, if the mean firing rate of the session was $<10 \mathrm{~Hz}$, and if the spatial information score was $>0.2$ (Table 1 ).

\section{Data analysis}

Description of basic firing properties

The amount of spatial information conveyed by a unit was measured by constructing a linearized firing-rate map (see Fig. 4C). For this purpose, position data from a behavioral session were scaled down by using a bin size of $4 \mathrm{~cm}^{2}$, and a raw spatial rate map was constructed by dividing the number of spikes by the duration of a visit for each bin. Spatial information was computed using the following equation (Skaggs et al., 1993):

$$
\text { Spatial information }=\sum_{i} p_{i} \frac{\lambda_{i}}{\lambda} \log _{2} \frac{\lambda_{i}}{\lambda}(\text { bit } / \text { spike })
$$

where $i$ denotes the bin, $p_{i}$ is the occupancy rate of the $i$ th bin, $\lambda i$ is the mean firing rate of the $i$ th bin, and $\lambda$ is the overall mean firing rate. The mean firing rate of a unit was calculated by averaging the firing rates in the raw rate map. A burst index was defined as the power of autocorrelation during the $1-6 \mathrm{~ms}$ period normalized by the power during the 1$20 \mathrm{~ms}$ period.

Definition of the boundaries of a place field and its center A place field was defined as three or more contiguous bins with firing rates $>20 \%$ of the peak firing rate of the unit. Only CA3 units with both 
the start and end bins identified in a linearized firing rate map were included in the analysis for place fields (see Fig. 4C). The mean firing rate used to calculate the rate modulation index was measured only within the boundaries of the place field of the unit.

\section{Rate modulation analysis}

To quantify the changes in discharge patterns of single units across different scene stimuli, a rate modulation index (RMI) was calculated using the mean firing rate of the place field for each condition. To directly compare the degrees of rate modulation during different sessions, the difference in firing rate between the left and right response-associated scene conditions was calculated as:

$$
\mathrm{RMI}=\left|\frac{\operatorname{mean}\left(\mathrm{FR}_{1}\right)-\operatorname{mean}\left(\mathrm{FR}_{2}\right)}{\operatorname{mean}\left(\mathrm{FR}_{1}\right)+\operatorname{mean}\left(\mathrm{FR}_{2}\right)}\right|
$$

where $\mathrm{FR}_{1}$ and $\mathrm{FR}_{2}$ denote in-field firing rates of the trials associated with the two conditions.

The rate modulation patterns across different levels of visual alterations for the scenes in the Blurred, Masked, and Overlay sessions were evaluated by quantifying the relationships between the mean firing rates and the levels of manipulations associated with visual scene stimuli, with these relationships visualized using a tuning curve. Because of a smaller number of correct trials observed in scene stimuli with higher difficulty levels, the mean firing rates for the last two difficulty levels in the Blurred and Masked sessions were combined (L50 and L70 into L57, R50, and R70 into R57 in the Blurred session; L3 and L4 into L34, R3, and R4 into R34 in the Masked session). The mean firing rates for eight conditions (two scenes $\times$ four ambiguity levels) were normalized from 0 to 1 , using the following equation:

$$
\text { Normalized } \mathrm{FR}_{\text {cond }}=\frac{\mathrm{FR}_{\text {cond }}-\min \left(\mathrm{FR}_{1-8}\right)}{\max \left(\mathrm{FR}_{1-8}\right)-\min \left(\mathrm{FR}_{1-8}\right)},
$$

where Normalized $\mathrm{FR}_{\text {cond }}$ is the normalized firing rate for a condition (cond), $\mathrm{FR}_{\text {cond }}$ denotes the mean firing rate for a condition, and min $\left(\mathrm{FR}_{1-8}\right)$ and $\max \left(\mathrm{FR}_{1-8}\right)$ denote the minimum and maximum values among the eight mean firing rates from each condition, respectively. For visualization, normalized firing rates were oriented so that the scene category associated with the higher firing rates were always on the righthand side of the graph.

The tuning curve was produced by fitting the data with the following set of model equations:

Quadratic model: $\alpha+\beta$ (scene) $+\gamma(\text { scene })^{2}$

and four-parameter sigmoidal model:

$$
F(x)=L+\frac{(U-L)}{1+\left(\frac{\text { scene }}{\alpha}\right)^{\beta}},
$$

where $L$ and $U$ are the lower and upper asymptotes, respectively, scene denotes the level of manipulation for the scene stimulus, and $\alpha$ and $\beta$ are the inflection point and slope at that point, respectively, of the sigmoidal curve (see Fig. 5A). The quadratic model and sigmoidal model were compared using Bayesian information criterion (BIC), where the model with the lower BIC value was defined as the best one to explain the unit data (Schwarz, 1978). The model with a better fit was displayed in a solid line along with its coefficient of determination. The two models were compared with each other to identify the single units that exhibited a categorical response near the optimal decision point (defined by the task demand). Specifically, if a unit showed a categorical response across different levels of ambiguity, the neural activity should be better explained by the sigmoidal model than by the quadratic model (see Fig. $5 A$ ). However, if a unit exhibited gradual changes, it should be fit better by the quadratic model than by the sigmoidal model (see Fig. $5 B$ ). The proportions of units better explained by the sigmoidal model were compared in the control and DG-lesion groups using $\chi^{2}$ tests for within-session comparisons and $z$-score tests for comparing across different sessions. This analytical strategy was previously used to successfully differentiate functional classes of cells in the perirhinal cortex during an object recognition task (Ahn and Lee, 2017).

In addition, the linear model and the three-parameter sigmoidal model, which are reduced forms of the quadratic model and the sigmoidal model, were also used for the scene-tuning curve estimation. The linear model is identical to the quadratic model, except that the third term $(\gamma)$ was removed. For the three-parameter sigmoidal model, the upper and lower asymptotes were combined as a single parameter.

To assess the tuning curve on a population level, the normalized mean firing rates of each unit were reoriented based on the sign of the slope of the better fitted model in the individual model fitting results. Reoriented normalized mean firing rates were averaged for each of eight levels, separately for the control and lesion groups, to produce the population mean firing rate for each scene category. Estimation of the scenetuning curve for the population data were performed in the same manner as for individual units, as described above.

\section{Experimental design and statistical analysis}

Behavioral performance from all recording sessions (Familiar, Blurred, Overlay, Masked, and Novel) was analyzed using a three-way repeatedmeasures ANOVA (group, session type, and day) with the rat identity nested within the group factor, implemented in JMP 11 statistical software package (SAS Institute). The results of post-hoc $t$ tests for ANOVA tests (Fig. 1D) comparing session type and group were corrected using a Bonferroni correction procedure for multiple-comparison corrections. For the Blurred, Overlay, and Masked sessions, a two-way repeatedmeasures ANOVA (group, ambiguity level) with the rat identity nested within the group factor was used (Fig. $1 E-G$ ). The post-hoc $t$ tests for comparing ambiguity levels and groups were corrected using Bonferroni correction procedures for multiple comparisons. Unless stated otherwise, differences in unit-firing properties were compared by KolmogorovSmirnov tests (K-S test), with the level of statistical significance set at $\alpha=$ 0.05 , implemented in StatView software package (SAS Institute). $\chi^{2}$ tests for independence, implemented in JMP 11, was used for comparing the proportions between the groups within a session. $z$-score tests were used for comparing two proportions associated with different sessions. A two-way ANOVA (group and session type as factors) were run in JMP 11 to compare the difference in the coefficient of determinations $\left(R^{2}\right)$ from the sigmoidal model fit. The results of post-hoc $t$ tests for ANOVA (see Fig. $9 E$ ) comparing session type and group were corrected using a Bonferroni correction procedure for multiple comparisons.

\section{Results}

\section{Lesions in the DG impair the ability of rats to disambiguate visual scenes}

Four rats each in the control group and DG-lesion groups were first tested with unaltered, familiar visual scenes (Fig. 1B), followed by the Blurred, Overlay, and Masked sessions (Fig. 1C). Compared with controls, DG lesions affected performance differently, depending on the type of stimulus manipulations. Three-way repeated-measures ANOVA, with group, session type, and day as factors, revealed a significant main effect of session type $\left(F_{(4,53.08)}=11.045, p=0.001\right)$ and a significant interaction between group and session type $\left(F_{(4,53.08)}=4.16, p=0.005\right)$. The main effect for group $\left(F_{(1,12.21)}=3.61, p=0.081\right)$ or day $\left(F_{(1,53.17)}=0.038, p=0.845\right)$ and interaction between group and day $\left(F_{(1,53.17)}=0.174, p=0.678\right)$, session and day $\left(F_{(4,53.17)}=2.49\right.$, $p=0.054)$, and the three-way interaction $\left(F_{(4,53.17)}=1.66\right.$, $p=0.173$ ) were not significant. When the original scenes were used as stimuli in the Familiar session, the control and DGlesion groups performed similarly, $\sim 90 \%\left(t_{(53.27)}=0.09\right.$, $p=0.249$, Bonferroni-corrected; Fig. 1D, Familiar). However, when visual scenes were made ambiguous in the Blurred session, rats with DG lesions showed a significant reduction in response accuracy compared with controls $\left(t_{(53.27)}=-2.68, p=0.01\right.$, 
A Control DG lesion
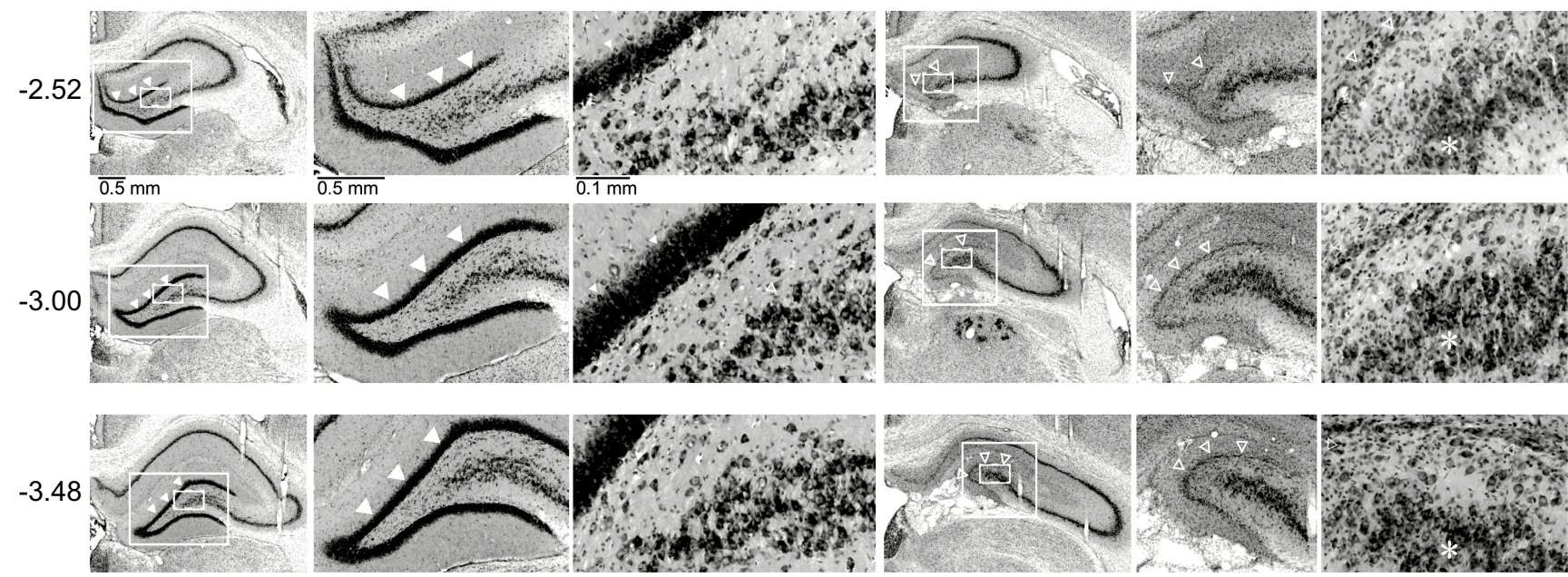

B

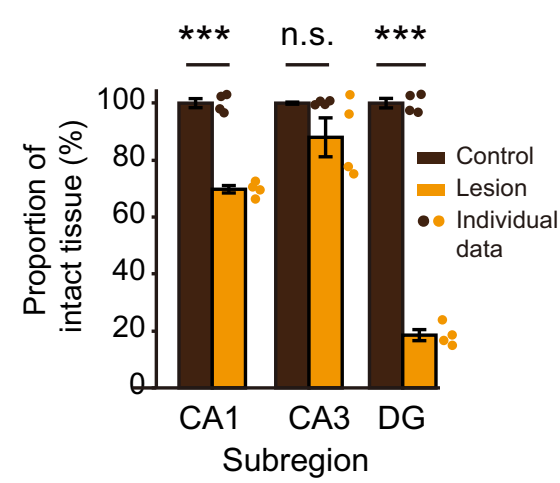

C

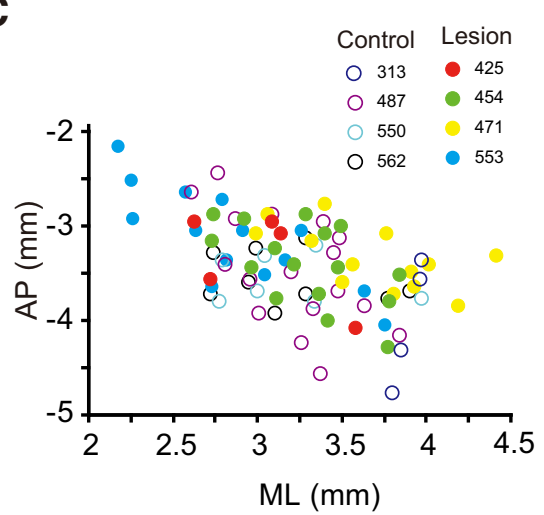

D

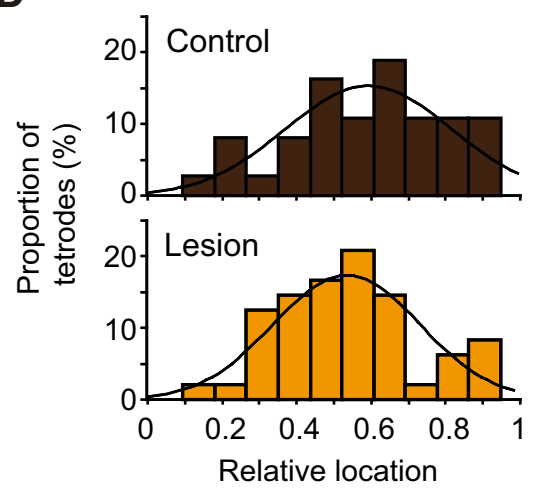

Figure 2. Histological verification of DG lesions. $A$, Examples of thionin-stained sections from the control and DG-lesion groups. For each hippocampal section (4× magnification, left), the DG/hilar area (larger rectangle) was zoomed and shown as a magnified image (10× magnification, middle). The suprapyramidal blade of the DG and the hilar area (smaller rectangle) was further zoomed to show changes observed in the DG granule cell layer and CA3 pyramidal cell layer (40× magnification, right). Solid and open arrowheads indicate intact and damaged granule cell layers in the control and lesion groups, respectively. Asterisks in the DG-lesion group denote the relatively intact pyramidal cells in the CA3. Scale bars are shown below the sections. AP coordinates (distances from bregma) are given at the left bottom corners of the sections. $B$, The extent of the lesion in the hippocampal subregions based on 3D volumetry. Proportions of intact tissue in individual rats are shown in colored dots next to the bar graphs. Values are given in mean \pm SE. n.s. not significant, ${ }^{* * *} p<0.001$. C, Distribution of the AP and ML coordinates of tetrodes. Tetrodes from control rats are depicted as open circles, and those of the DG-lesioned rats are as filled circles. Individual rats are shown in different colors. $\mathbf{D}$, Distribution of the relative locations of the tetrodes in the control and DG-lesion groups. The relative location of 1 denotes the boundary between CA3 and CA2. The black curves denote estimated normal distributions.

Bonferroni-corrected post-hoc $t$ test; Fig. 1D, Blurred). In the Masked sessions, both groups showed $\sim 85 \%$ accurate responses, with no significant difference between the control and DG-lesion groups $\left(t_{(53.27)}=-1.42, p=0.35\right.$, Bonferroni-corrected post-hoc $t$ test; Fig. 1D, Masked). Behavioral performance levels were similar between two groups during the Overlay sessions $\left(t_{(53.27)}=1.65\right.$, $p=0.10$, Bonferroni-corrected post-hoc $t$ test; Fig. 1D, Overlay).

The accuracy of choices made by the control and DG-lesion groups across different ambiguity levels during the Blurred, Masked, and Overlay sessions were compared by two-way repeated-measures ANOVA, with group and ambiguity level as factors. In the Blurred session, the results showed a significant interaction between group and ambiguity level $\left(F_{(4,64)}=3.901\right.$, $p=0.006)$. Compared with control rats, rats with DG lesions showed significant impairment in response accuracy at ambiguity levels of $\geq 40 \quad\left(t_{(68)}=-2.22, p=0.015 ; t_{(68)}=-3.87\right.$, $p=0.0001$; and $t_{(68)}=-3.38, p=0.001$, respectively, for 40,50 , and 70 conditions, Bonferroni-corrected post-hoc $t$ test; Fig. $1 E)$, but not at ambiguity levels of $<40\left(t_{(68)}=0.35, p=0.723\right.$; and $t_{(68)}=-0.58, p=0.28$, respectively, for 0 and 30 conditions, Bonferroni-corrected post-hoc $t$ test). However, in the Masked
(Fig. 1F) and Overlay (Fig. 1G) sessions, the main effect of ambiguity level was significant (Masked: $F_{(4,64)}=10.128, p=0.001$; Overlay: $\left.F_{(3,43)}=4.746, p=0.01\right)$ with no significant group effect (Masked: $F_{(1,68.83)}=0.013, p=0.91 ;$ Overlay: $F_{(1,43.18)}=0.003$, $p=0.956)$ and no significant interaction effect as well (Masked: $F_{(4,64)}=0.947, p=0.442$; Overlay: $\left.F_{(3,43)}=0.178, p=0.911\right)$.

These findings strongly suggest that the DG plays an important role in recognizing ambiguously blurred versions of the familiar visual scenes, but not when the visual scenes are unaltered in other ways. Because the performance of the control and lesion groups did not differ significantly from each other when the visual scenes were blurred relatively mildly (i.e., $30 \%$ ), it is likely that the DG lesions affected visual pattern separation when substantial noise (i.e., $\geq 40 \%$ Gaussian blur) was added to the original scene stimuli.

\section{Histological verification of colchicine-induced lesions}

Injection of colchicine into the dorsal DG ablated the granule cell layer and the hilar region, while leaving the CA3 pyramidal cell layer relatively intact (Fig. $2 A$ ). Compared with the aCSFinjected controls (Fig. 2B; effect of interaction: $F_{(2,12)}=91.08$, $p=0.001$ ), the rats with colchicine injection showed almost 
complete damage $\left(\sim 85 \%, t_{(15.88)}=\right.$ $-18.59, p=0.001$, Bonferroni corrected) of the DG granule cell layer. The CA1 subregion also showed considerable damage $\left(\sim 35 \%, t_{(15.88)}=\right.$ $-6.90, p=0.001$, Bonferroni corrected), but there was no significant difference between control and lesion groups in the CA3 $\left(t_{(15.88)}=\right.$ $-2.74, \quad p=0.22$, Bonferroni corrected). The extent of damage observed in this study was consistent with those reported in the previous studies (McNaughton et al., 1989; Lee and Kesner, 2004; Ahn and Lee, 2014; Sasaki et al., 2018).

To assess whether there were any differences in tetrode tracks between two groups, we identified antero-posterior (AP) and mediolateral (ML) coordinates of all of the tetrodes in which any unit was recorded during the entire session (Fig. 2C). There was no significant difference between two groups in both $\mathrm{AP}$ and ML coordinate distributions (AP: $\chi_{(2)}^{2}=6.624, p=0.073$, ML: $\chi_{(2)}^{2}=1.989, p=0.74, \mathrm{~K}-\mathrm{S}$ test). Similarly, there was no significant difference between the two groups in the relative recording locations $\left(\chi_{(2)}^{2}=\right.$ 4.764, $p=0.185$; Fig. $2 D$ ). This result indicates that the differences found between two groups were unlikely to be caused by the functional heterogeneity along the proximodistal axis of the CA3 (Lee et al., 2015).

\section{Basic firing properties were unaffected by DG lesions}

Despite the absence of DG inputs, there was no significant difference in raw spike waveforms (Fig. $3 A$ ). Spike width $\left(\chi_{(2)}^{2}=\right.$ $5.45=, p=0.131$, K-S test $)$, burst index $\left(\chi_{(2)}^{2}=4.29, p=0.233, \mathrm{~K}-\right.$ $\mathrm{S}$ test), mean firing rates $\left(\chi_{(2)}^{2}=3.84, p=0.293\right.$, K-S test $)$, and peak firing rates $\left(\chi_{(2)}^{2}=6.61, p=0.073\right.$, K-S test $)$ in CA3 units recorded during the Familiar sessions did not differ significantly between the lesion and control groups (Fig. $3 B$ ). Intact place cells were observed in the CA3 during the VSM task in both the control group (Fig. 4A) and the DG-lesion group (Fig. 4B), which is consistent with the previous studies reporting intact spatial selectivity of CA3 cells after the DG lesion (McNaughton et al., 1989).

\section{Categorical rate modulation in the CA3 across ambiguous scenes occurs in the control, but not the in the DG-lesion group}

Pattern separation is a computational process that reduces the amount of overlap in output patterns using similar input patterns (O'Reilly and McClelland, 1994; Treves and Rolls, 1994). To test whether pattern separation occurred in the CA3 for ambiguous visual scenes in the VSM task, the neuronal firing rates for ambiguous scenes, including the originals, were calculated from the place fields defined on a linearized rate map (Fig. 4C). For each unit, the firing rates across different scene stimuli were normalized and the difference (RMI between the firing rates for the left and right choice-associated scenes) was determined. Although the degree of rate modulation between left and right scenes was similar in the Familiar session for both groups $(z=$ $-0.05, p=0.960$, Mann-Whitney $U$ test; Fig. $4 D$ ), and rate modulation was lower in the DG-lesion group when ambiguity was introduced in the scene stimuli during the Blurred sessions $(z=$
$-2.04, p=0.041$, Mann-Whitney $U$ test). However, the group differences in rate modulation were not observed during the Masked $(z=1.02, p=0.307$, Mann-Whitney $U$ test $)$ or Overlay sessions $(z=$ $-0.411, p=0.681$, Mann-Whitney $U$ test).

Firing-rate tuning curves for visual scenes were subsequently determined for individual CA3 cells. Place cells in the CA3 showing a categorical tuning curve across the blurred scene stimuli according to their choice contingency were operationally defined as pattern separators (Fig. $5 \mathrm{~A}$ ). In contrast, place cells in the CA3 showing a gradual change in firing rates across the blurred scenes were regarded as non-pattern separators (Fig. $5 B$ ). To objectively determine the categorical nature of neuronal firing for the scene stimuli, the normalized firing rates of a cell were fitted using two models [i.e., a sigmoidal model for fitting stepwise (or categorical) data (Fig. 5A), and a quadratic model for fitting gradually changing data (Fig. $5 B$ )]. The model that better fits the data was regarded as the tuning curve of the cell.

To test the validity of the unit-categorizing procedures described above, we first fit all units $(N=179)$ from three session types (Blurred, Masked, and Overlay) using the sigmoidal model (including the ones from the units better fit by the quadratic models) to quantify the similarity between the two scene representations coded by the neural states (measured by the median) above and below the inflection point (Fig. 5A). The distance between the neural states was larger in the pattern separators than in the non-pattern separators, meaning that the pattern separating neurons coded ambiguous stimuli into more discrete states $\left(\chi_{(2)}^{2}=32.754, p=0.001\right.$, K-S test; Fig. $\left.5 C\right)$. The procedures for unit categorization seem valid because the units whose tuning curves for scenes were fit better by the sigmoidal models (i.e., pattern separators) showed tighter fitting than those fit by the quadratic models (i.e., non-pattern separators; $\chi_{(2)}^{2}=53.175$, $p=0.001, \mathrm{~K}-\mathrm{S}$ test). In addition, the inflection points of those units were located closer to the midpoint of the ambiguity levels $\left(\chi_{(2)}^{2}=27.281, p=0.001\right.$, K-S test; Fig. 5D). 
A

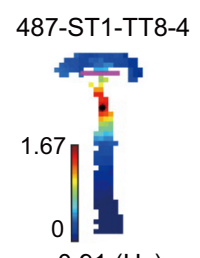

$0.91(\mathrm{~Hz})$

487-ST1-TT8-3

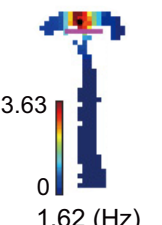

Control
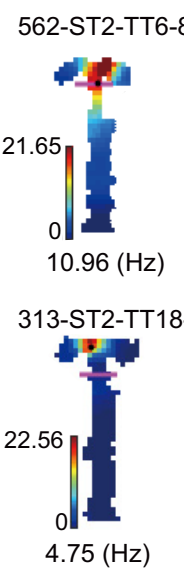

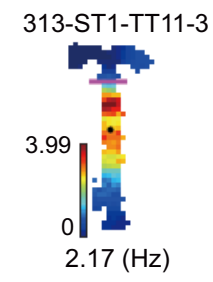

$2.17(\mathrm{~Hz})$
B

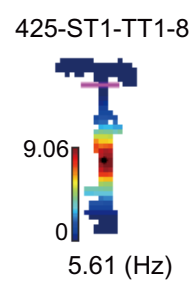

DG lesion

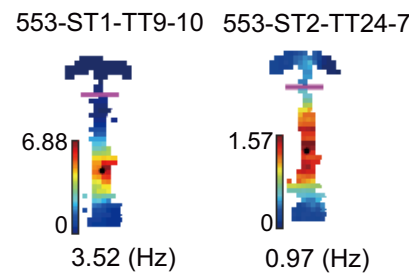

C

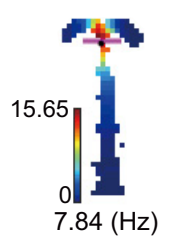

454-ST2-TT6-2

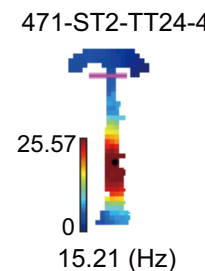

553-ST1-TT12-8
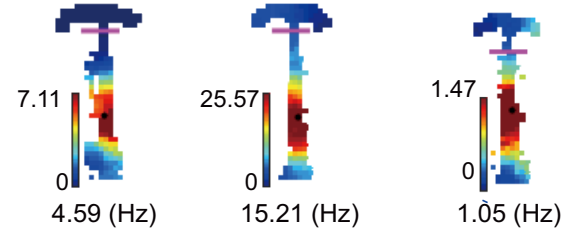

D
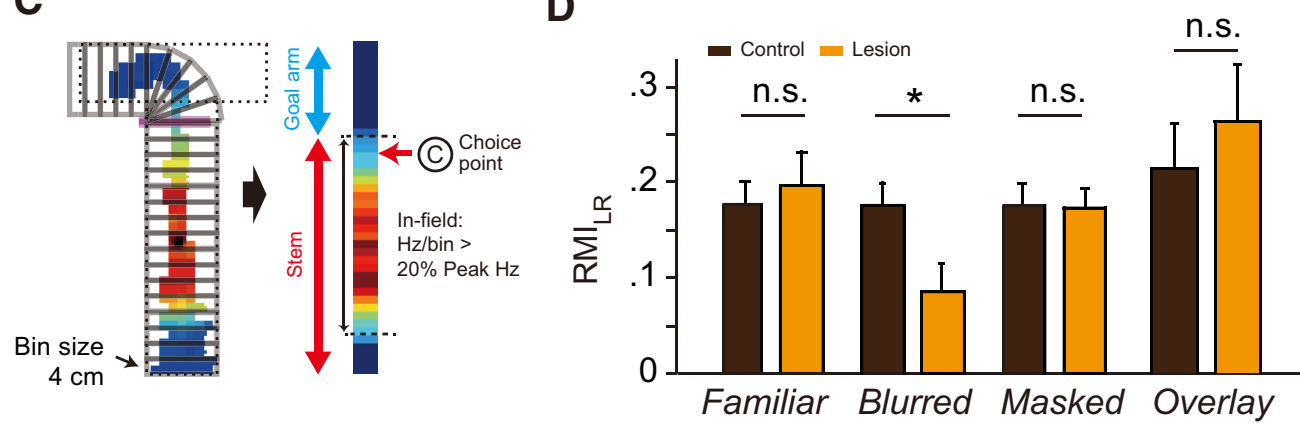

Figure 4. Scene-dependent rate modulation in the CA3 units. $A$, Examples of spatial firing patterns of CA3 units with intact DG. Cell identities are marked on top of the unit examples, which denote information about the rat, session, tetrode, and cluster identity of each unit in that order. Numbers on the top of the color bar indicate a peak firing rate. In-field mean firing rates are shown below each spatial firing rate map. Black dots indicate the center of mass of the place field. $\boldsymbol{B}$, Examples of spatial firing patterns of CA3 units from rats with DG lesions. $\boldsymbol{C}$, Illustration of the linearization process of a CA3 place field. The choice point was defined as the bin in which the trajectories for the left and right trials differed significantly for at least two consecutive bins. $\mathbf{D}$, Rate modulation index (RMI) between the left and right response-associated scenes in the Familiar, Blurred, Masked, and Overlay sessions. Values are given in the mean \pm SE. n.s. not significant, ${ }^{*} p<0.05$.

We found that the scene-related firing patterns of the CA3 cells in the control group were better fit by the sigmoidal model than the quadratic model (Fig. 6A), and vice versa for the lesion group (Fig. $6 B$ ). That is, pattern separators were more frequently found in the control group than in the lesion group and vice versa for non-pattern separators.

The goodness-of-fit for the sigmoidal model (measured by the coefficient of determination) was significantly higher on average in the control group ( $N=24$ cells) than in the lesion group $(N=16$ cells $)$ in Blurred sessions $\left(\chi_{(2)}^{2}=9.60, p=0.016\right.$, K-S test; Fig. $6 \mathrm{C}$ ). The inflection points were also distributed closer to the optimal decision point in the control group than in the lesion group $\left(\chi_{(2)}^{2}=8.817, p<0.05\right.$, K-S test; Fig. $\left.6 C\right)$. The proportion of pattern separator-like cells was significantly lower in the DGlesion than in the control group in the Blurred session $\left(\chi_{(1)}^{2}=\right.$ 5.934, $p=0.015, \chi^{2}$ test; Fig. $\left.6 D\right)$. The attenuation of pattern separator-like activity was also observed at a population level. That is, the population tuning curve for visual scene in the DGlesion group was similarly explained by the sigmoidal model $(\mathrm{BIC}=-15.12)$ and quadratic model $(\mathrm{BIC}=-14.59)$, whereas the sigmoidal model explained the neural firing more powerfully in the control group $(\mathrm{BIC}=-26.62$ for sigmoidal model; $\mathrm{BIC}=$ -23.29 for quadratic model; Fig. $6 E$ ). These findings suggest that the behavioral impairment observed in rats with DG lesions (Fig. $1 D, E)$ may be related to the degradation of discrete memory representations for similar visual scenes.

It is unlikely that the above-mentioned differences between the groups were caused by the inherent differences in firing rates that might be better suited for either model fitting because the place cells of the two groups in Blurred sessions ( $N=40$ cells) were similar to each other with respect to the in-field mean firing rate $\left(\chi_{(2)}^{2}=3.267, p=0.39\right.$, K-S test $)$, normalized firing rate $\left(\chi_{(2)}^{2}=6.667, p=0.071, \mathrm{~K}-\mathrm{S}\right.$ test $)$, and the $\mathrm{SD}$ of the normalized firing rate $\left(\chi_{(2)}^{2}=3.28, p=0.39\right.$, K-S test; Fig. $\left.7 A\right)$. To equate the parameters between the models, we also tried a reduced sigmoidal model and a linear model to estimate the scene-tuning curve. The reduced sigmoidal model was intended to match the number of parameters to that of the quadratic model. However, consolidating the upper and lower asymptotes to one parameter resulted in nonoptimal fitting results in multiple units (Fig. $7 B$ ). For example, when estimating the scene-tuning curve of a unit, the reduced model showed the inflection point near the first level, followed by a steep negative slope, which clearly deviated from the scene stimuli-associated firing rates (Fig. $7 C$ ).

\section{Categorical firing patterns in the CA3 for visually masked or mixed scene stimuli are not affected by DG lesions}

To determine whether changes in the visual scene could cause the degradation of rate modulation in the CA3 after DG lesions, data from the Masked sessions, in which visual scenes were partially occluded but the patterns were still visible, were analyzed. The performance of the two groups did not differ significantly during the Masked sessions (Fig. 1B,F), suggesting that partial occlusion of the visual scene does not affect pattern separation in the $\mathrm{CA} 3$ as much as does blurring of the scene. 

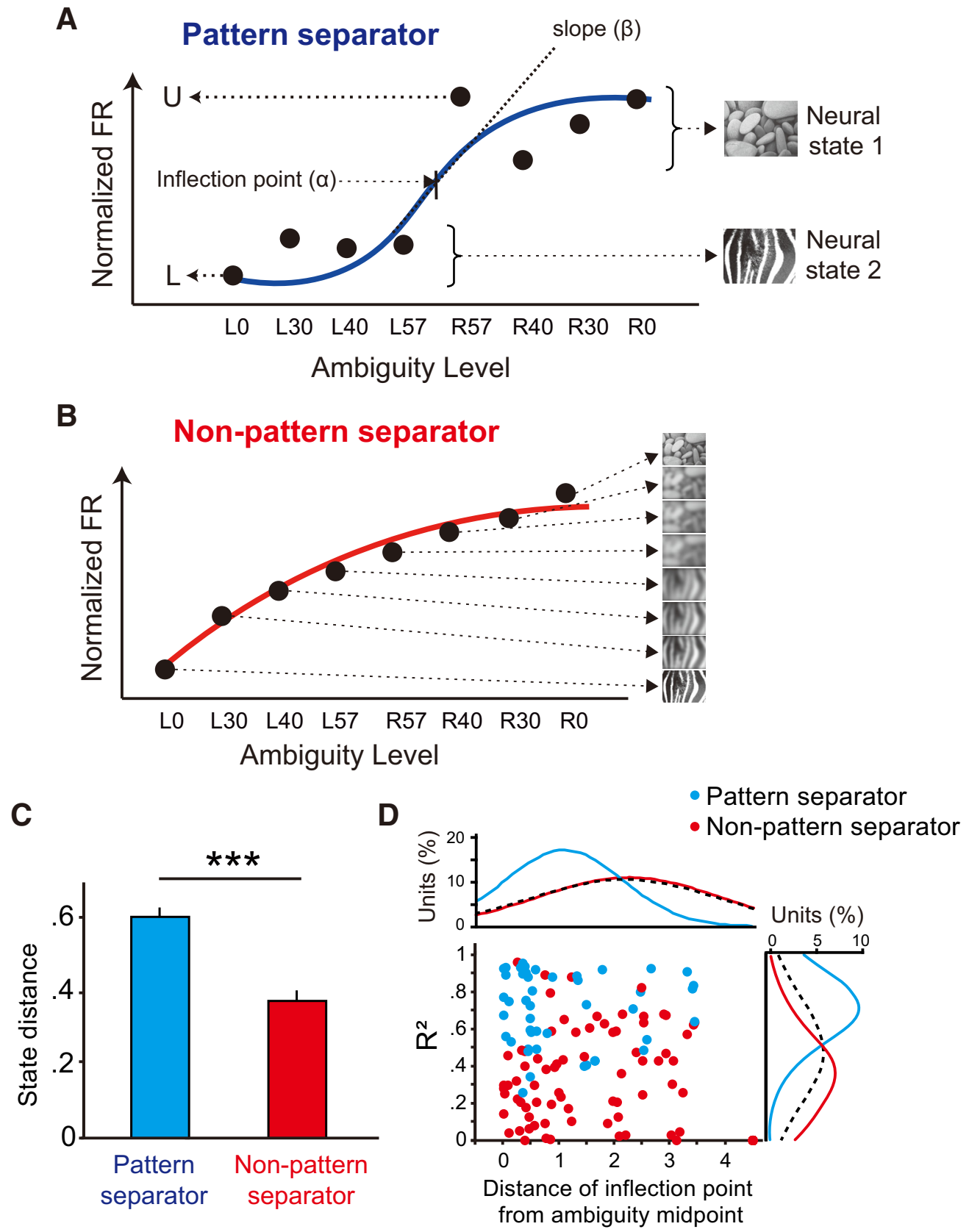

Figure 5. Sigmoidal and quadratic model estimation for scene-tuning curves in the CA3 units. $A$, Illustration of the four-parameter sigmoidal model used to evaluate the stepwise and categorical rate modulation across ambiguous visual scenes. The "neural state" denotes the median normalized firing rate (FR) of the points above and below the inflection point. U, Upper asymptote; L, lower asymptote. $\boldsymbol{B}$, Illustration of the quadratic model used to evaluate the gradual rate modulation across ambiguous visual scenes. $\boldsymbol{C}$, State distance (i.e., Fig. $5 \mathrm{~A}$, the distance between neural state 1 and 2) between two categorical responses of the pattern separator and non-pattern separator cells from the Blurred, Masked, and Overlay sessions. Values are given in mean \pm SE. ${ }^{* * *} p<0.001$. D, Scatterplot showing the correlation between the coefficient of determination $\left(R^{2}\right)$, and the distance of the inflection point from the midpoint of the stimulus ambiguity levels from the Blurred, Masked, and Overlay sessions ( $N=179$ cells). The unit types defined by the BIC comparisons are depicted in different colors. The percentage unit distribution on the right-hand side shows the $R^{2}$ distributions for the pattern separators (blue), non-pattern separators (red), and all cells together without any functional categorization (black dotted line). The percentage of unit distribution on the top shows the distance distributions for the pattern separators (blue) and non-pattern separators (red). The distribution for all cells without any functional categorization is also overlaid (black dotted line).

Consistent with these behavioral results, the pattern separator-like cells were equally abundant in the DG-lesion (Fig. $8 B$ ) and control (Fig. $8 A$ ) groups during the Masked sessions $\left(\chi_{(1)}^{2}=\right.$ $0.004, p=0.949, \chi^{2}$ test). Similarly, the percentages of pattern separator-like $(44.8 \%)$ and non-pattern separator-like (55.2\%) cells were similar in the DG-lesion group $(z=-0.79, p=0.429$, $z$-score test; Fig. $8 C$ ). In addition, the proportion of pattern separator-like cells in the CA3 of the DG-lesion group during Masked sessions (44.8\%) was similar to that of the control group (50.9\%) in the Masked ( $z=-0.53, p=0.596, z$-score test) and Blurred ( $z=-0.37, p=0.703, z$-score test) sessions, but was significantly higher than that of the DG-lesion group during Blurred sessions ( $z=1.75, p=0.04, z$-score test). The population tuning curves for visual scenes were similar between the two 
A
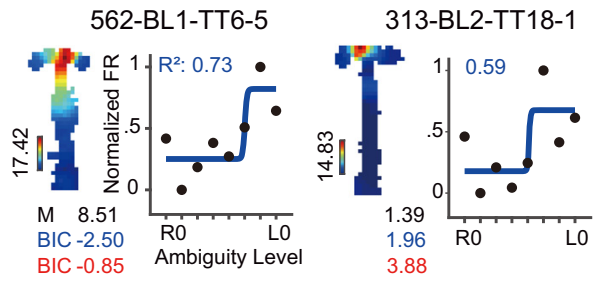

313-BL1-TT3-6
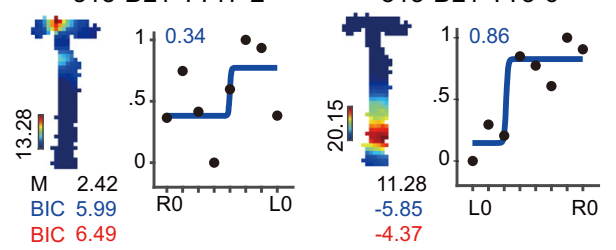

562-BL 2-TT3-6

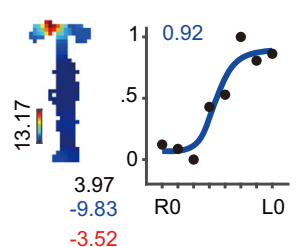

562-BL1-TT4-4

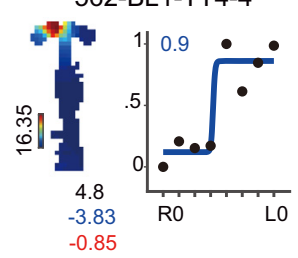

562-BL1-TT6-7

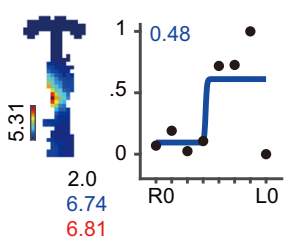

B

553-BL2-TT24-3

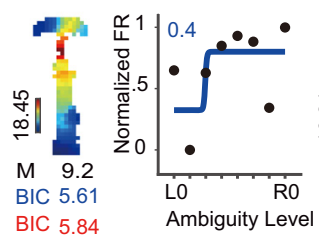

C

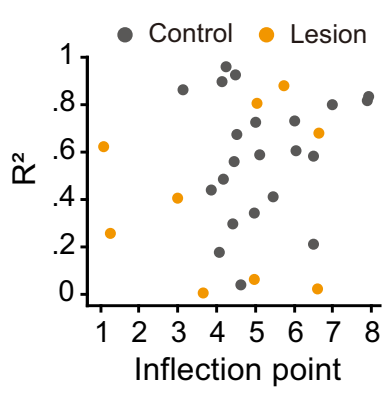

425-BL1-TT1-4

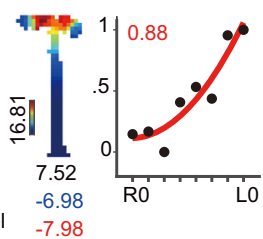

553-BL1-TT10-4

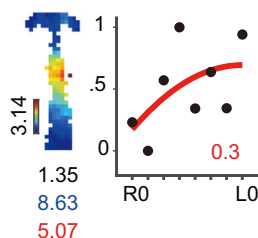

D

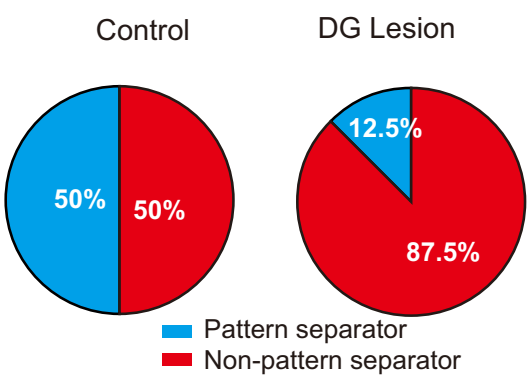

487-BL1-TT8-1

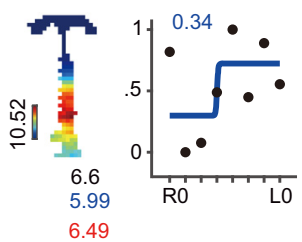

487-BL1-TT8-6

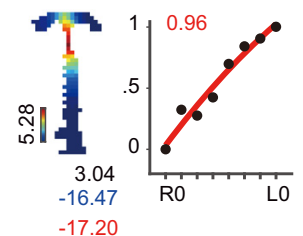

Figure 6. Disrupted categorical rate modulation in the CA3 with DG lesions. $A$, Examples of stepwise rate modulation in the CA3 units with intact DG. M denotes the in-field mean firing rate, $R^{2}$ denotes the goodness-of-fit of the better explaining model. BIC values (blue from the sigmoidal model and red from the quadratic model) used for the categorization are shown below the rate map. Blue lines indicate well fit sigmoidal models, and red lines indicate well fit quadratic models. $B$, Examples of gradual rate modulation in the CA3 units with DG lesions. The dotted lines indicate poor fit $\left(\mathrm{R}^{2}<0.3\right)$ of the models. $C$, Scatterplot of the inflection points and the goodness-of-fit parameters from the sigmoidal model in $B /$ urred sessions $(N=40$ cells). The control and lesion groups are marked in different colors. Units with inflection points $<1$ were excluded from the plot ( 7 units from the lesion group and 2 units from the control group). $\boldsymbol{D}$, The proportions of units that are better explained by the sigmoidal model (pattern separator-like cells, blue) and by the quadratic model (non-pattern separators, red) in the control and lesion groups. $\boldsymbol{E}$, The population scene-tuning curve from control (left) and DG-lesion group (right) in the Blurred sessions. BIC values from the sigmoidal model (blue) and quadratic model (red) are shown below the scene-tuning curves (blue solid line: sigmoidal, red dashed line: quadratic).

groups in Masked sessions (Fig. 8D). The sigmoidal model explained neural firing more powerfully than the quadratic model did in the control group (BIC $=-27.81$ for sigmoidal model; $\mathrm{BIC}=-21.65$ for quadratic model), as well as in the DGlesion group $(\mathrm{BIC}=-25.38$ for sigmoidal model; $\mathrm{BIC}=-22.35$ for quadratic model).

Similar to the Masked session, performance in the Overlay session was not affected by DG lesions. During the Overlay session, pattern separator-like cells were as frequently found in the DG-lesion group (Fig. 9B) as in the control group (Fig. 9A; $\chi_{(1)}^{2}=0.003, p=0.957, \chi^{2}$ test; Fig. $\left.9 C\right)$. Similarly, the population-tuning curves for visual scenes were similar between the two groups in Overlay sessions (Fig. 9D). The sigmoidal model explained the neural firing more powerfully than the quadratic model did in the control group $(\mathrm{BIC}=-28.82$ for sigmoidal model; $\mathrm{BIC}=-14.23$ for quadratic model), as well as in the DGlesion group $(\mathrm{BIC}=-21.57$ for sigmoidal model; $\mathrm{BIC}=-16.09$ for quadratic model). Comparing the goodness-of-fit of the sigmoidal model across three session types revealed a significant interaction between the group and session type factors $\left(F_{(2,173)}=\right.$ $3.904, p=0.022$, two-way ANOVA) only during the Blurred session $\left(t_{(173)}=-3.401, p=0.001\right.$, Bonferroni corrected $)$, but not in the Masked session $\left(t_{(173)}=-1.241, p=0.216\right.$, Bonferroni corrected) or Overlay session $\left(t_{(173)}=0.20, p=0.842\right.$, Bonferroni corrected; Fig. 9E).

These findings indicate that impaired performance of DGlesioned rats during Blurred sessions was not due to any visual changes in original familiar scenes. That is, altering the original visual scenes in such a way that rats could still see the overall patterns in the stimuli did not significantly disrupt performance as much as using blurred scenes. Moreover, the relatively robust stepwise tuning curves of the CA3 cells in the DG-lesioned rats may be the neural correlates of such robust performance in the lesion group. 


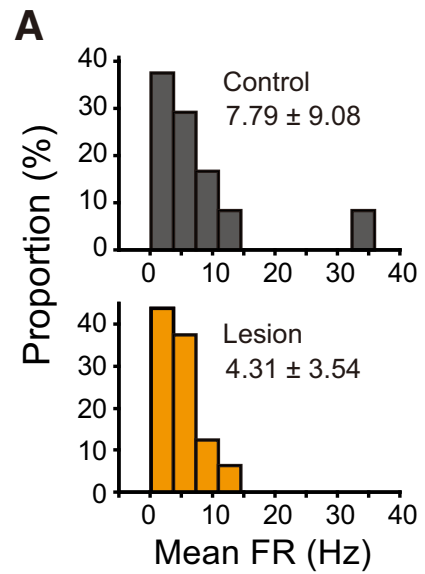

B

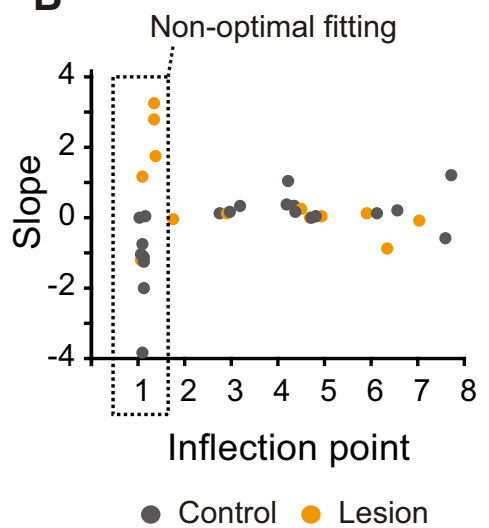

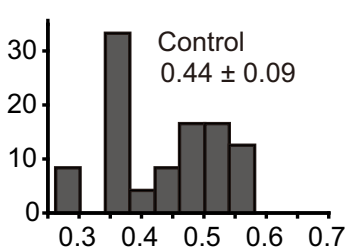
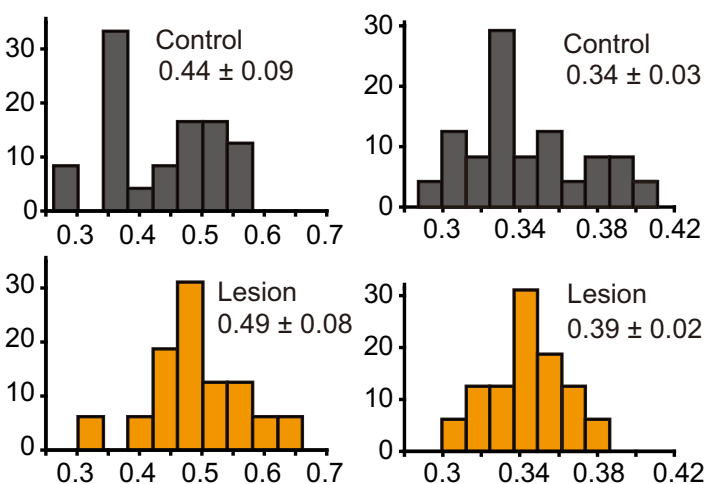

Normalized FR $(\mathrm{Hz})$

C

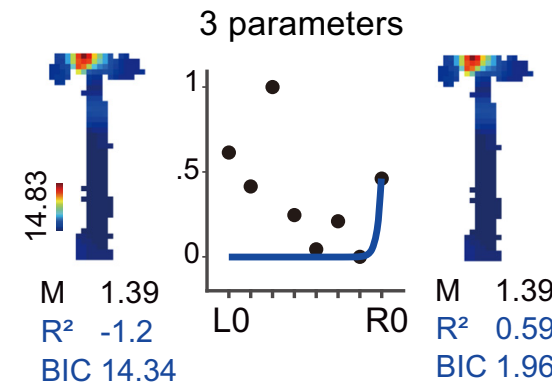

4 parameters

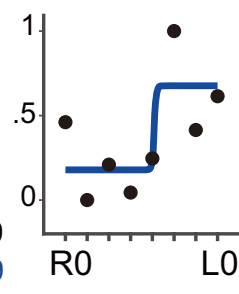

Figure 7. Alternative models for the categorical rate modulation in the CA3. $\boldsymbol{A}$, Distribution of the mean firing rates, normalized mean firing rates, and the SD of the normalized firing rates of place cells from Blurred sessions ( $N=40$ neurons). $B$, Scatterplot of the inflection points and the slope at those points, estimated by the reduced sigmoidal model using only a single parameter for both upper and lower asymptotes. The dotted box indicates the units showing the nonoptimal fitting. $\boldsymbol{C}$, Example of nonoptimal fit caused by the reduced model (left), shown along with the model fit result from the full sigmoidal model.

\section{Discussion}

The current study showed that rats with severe damage to the DG were impaired in processing ambiguous scenes, but not when the scenes were unaltered. Corroborating these behavioral results, this study found that scene-associated rate modulation in the CA3 was weaker in rats with DG lesions than in controls. Also, fewer pattern separator-like cells were present in the CA3 of rats with DG lesions compared to controls. In agreement with these neural and behavioral findings, we found that, in contrast to controls, place cells in the CA3 from the DG-lesion group were not concentrated at the choice point of the maze, a region strategically important for task demand. These neural and behavioral findings were specific to conditions in which the original scenes were made ambiguous by blurring, but not to conditions in which the original scenes were masked to allow rats to recognize the overall visual patterns in scene stimuli. In sum, these findings suggest that the DG plays critical roles in inducing pattern separation in the CA3 during memory retrieval when original scenes are made ambiguous.

Computational, behavioral and physiological studies have shown that the DG-CA3 networks are essential in pattern separation (Marr, 1971; O’Reilly and McClelland, 1994; Treves and Rolls, 1994; Gilbert et al., 2001; Leutgeb et al., 2007; Bakker et al., 2008; Lee and Solivan, 2010; Nakashiba et al., 2012; Ahn and Lee, 2014; Neunuebel and Knierim, 2014; GoodSmith et al., 2017; van Dijk and Fenton, 2018). However, behavioral evidence of pattern separation in the DG-CA3 networks was obtained predominantly using spatial memory tasks, in which rats were required to distinguish among similar spatial locations or environments (Gilbert et al., 2001; Nakashiba et al., 2012; van Dijk and Fenton, 2018). In contrast, most physiological studies have recorded cells in the DG or CA3 when rats forage for randomly scattered food, not during the performance of memory tasks. The results of our study may fill the gap between behavioral and physiological results, by showing that CA3 cells require the DG network to physiologically display the characteristics of the pattern-separating unit, including categorical and orthogonal coding of similar environmental stimuli. The absence of these pattern separatorlike CA3 units was observed in the DG-lesion group, and the lesion group's performance was impaired in Blurred sessions.

One of the models explaining the roles of the DG in facilitating pattern separation emphasizes its ability to provide sparse, yet powerful inputs via mossy fibers to a small subset of CA3 cells during the acquisition of memory (Treves and Rolls, 1994). The subset of cells chosen by the sparse inputs of the DG may form a critical node, resulting in a rich Hebbian association of environmental stimuli mostly fed via the perforant path from the entorhinal cortex. This model suggests that these memory representations in the CA3 network can be retrieved mostly by the cues provided via the perforant path and that the DG may not be involved in the retrieval of memory. Although these results may seem to disagree with the predictions made by these computational models, because DG lesions impaired our performance 
A

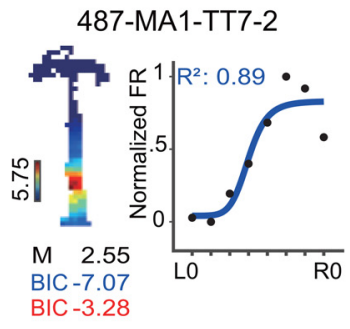

487-MA1-TT2-6

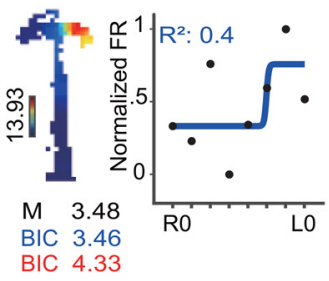

B

454-MA1-TT8-2

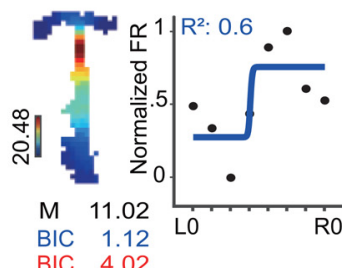

425-MA2-TT1-1

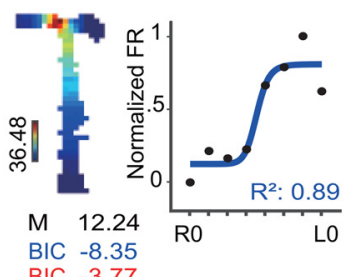

$\mathrm{BIC}-3.77$

C Control
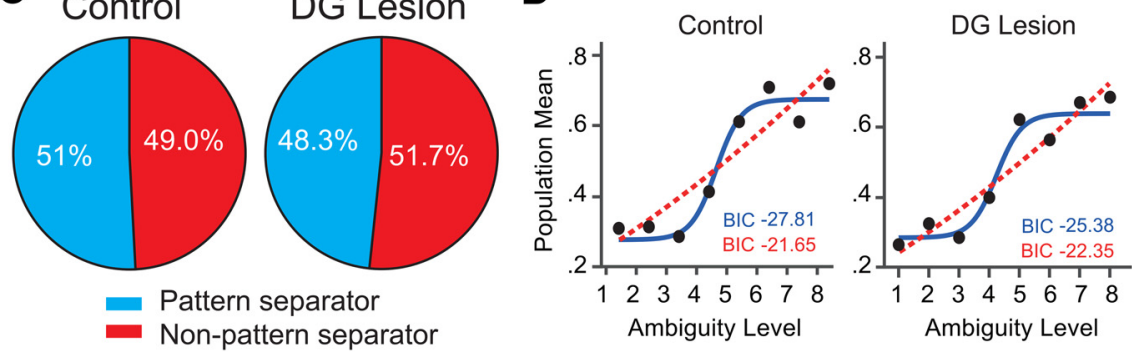

Figure 8. Scene-dependent rate modulation in the CA3 during retrieval of partially occluded visual scenes. $\boldsymbol{A}$, Examples of stepwise rate modulation in CA3 units with intact DG, as shown in Figure $6 \mathrm{~A}$. Blue lines indicate well fit sigmodal models. BIC values (blue from the sigmoidal model and red from the quadratic model) used for the categorization are shown below the rate map. $\boldsymbol{B}$, Examples of categorical rate modulation in CA3 units with DG lesions. $\boldsymbol{C}$, The proportion of units that were better explained by the sigmoidal model (pattern separator-like cells, blue) and by the quadratic model (non-pattern separator-like units, red) in the control and DG-lesion groups during the Masked sessions. $\boldsymbol{D}$, The population scene-tuning curve from control (left) and DG-lesion group (right) in the Masked sessions. BIC values from the sigmoidal model (blue) and quadratic model (red) are shown below the scene-tuning curve.

during Blurred sessions, performance deficits were not observed when the original visual scenes were unaltered, partially occluded or blurred slightly. The previous computational models simulated the retrieval mostly of the original representations from the CA3 using partial and/or noisy cues (O'Reilly and McClelland,
1994). The relatively intact performance of DG-lesioned rats under mildly blurred conditions in our study provides support for these computational models. Our results suggest that the DG is indispensable for memory retrieval when familiar environmental stimuli are significantly altered. Therefore, when environmental inputs are ambiguous, the mossy fiber-mediated inputs from the DG may carry some critical information for the CA3 network to access the original memory representations. Intact basic firing properties of CA3 cells in the DGlesioned rats when retrieving relatively familiar visual scenes suggest that the DG network is specifically activated when environmental ambiguity exceeds a certain threshold. Future studies should address the mechanism by which the DG network detects the level of ambiguity in the stimulus.

Consistent with the previous studies, there was some damage in the CA1 subfield as a result of the colchicine injection into the DG. It is possible that the degraded pattern separation of the single units in the CA3 in the DGlesion group might be attributable to the partial damage in the CA1. However, prior behavioral studies reported dissociations in performance with similar colchicine-based lesion techniques and with a similar amount of damage in the CA1 (Gilbert et al., 2001; Lee and Kesner, 2003). Furthermore, the CA3 receives major inputs from the DG, CA3, or entorhinal cortex, but not directly from the CA1 (Gonzales et al., 2001). Together, it is unlikely that the partial damage in the $\mathrm{CA} 1$ was the major cause of the degraded pattern separation in the DG-lesion group. Another concern for the DG-lesion group could be the absence of mossy cells in the hilar area in that group. Mossy cells in the hilar region may play key roles in providing feedback information to granule cells in the DG and bridging the CA3 and DG (Buckmaster et al., 1996). In addition, inactivating mossy cells induced hyperactivity in granule cells and impaired pattern separation (Jinde et al., 2012). Unfortunately, because colchicine eliminates granule cells and mossy cells altogether, it is impossible to determine the differential contributions of different cell types in the current study. Future studies with more cell type-selective methods are required to address these issues.

One of the key findings in the current study is that, unlike blurring the scenes, the partial occlusion of the visual scene 
stimulus (Masked session) enabled rats to perform the task without the DG, and that CA3 cells exhibited pattern separation normally without the DG. The neural mechanisms to explain why the DG is necessary only in recognizing the noisy stimuli (i. e., blurry scenes) but not for the partial stimuli (i.e., masked scenes) allow only speculations at this point. Although computational models assume that noisy (blurred) and partial (occluded) inputs might equally trigger pattern separation in the DG-CA3 networks (O'Reilly and McClelland, 1994), it is possible that noisy inputs might necessitate the DG functions more than partial ones for pattern separation in the CA3. Another possibility is that the extra testing period during which rats experienced the blurred and overlaid stimuli might have allowed the CA3 network to develop a new way to conduct pattern separation (or pattern completion) without the DG. One may think of a possibility that rats might have dealt with the masked stimuli as objects and used the perirhinal cortex to solve the task without the DG. Although that might explain why the DG lesions did not result in performance deficits, it cannot explain how the CA3 network still exhibited pattern separation without the DG. Furthermore, our masks appeared at pseudorandom locations of the screen on different trials, making it difficult for the rat to see the same local patterns across different masked trials.

Interestingly, the DG-lesioned rats were also minimally affected by the overlaying of two visual scene stimuli (Overlay session). The overlaying visual stimuli were intended to mimic the visual object morphing conditions (Ahn and Lee, 2017). It is possible that the scene overlay may have been qualitatively different from the object morphing. Visual stimuli with two scenes overlaying on each other with different opacity levels may have left structural cues for original scenes. The image analysis using the structural similarity index (Wang et al., 2004) suggested that overlaid scene stimuli were more similar to the original images compared with blurred or occluded scene stimuli. Future studies may use other ways of morphing two scenes that severely degrade the structural layout of the original scenes.

Recently, we have shown that neurons in the perirhinal cortex also exhibit pattern separator-like responses when rats were required to recognize ambiguously morphed images (Ahn and Lee, 2017). Although it would be ideal to record CA3 cells in the same object-recognition paradigm, there are several reasons that make such an attempt difficult. First, in rodents, it is often difficult to use the touchscreen-based recognition paradigm (Ahn and Lee, 2017) when physiologically recording neural activities from the hippocampus because principal neurons in the hippocampus are mostly place cells (especially in the dorsal hippocampus), meaning that they fire only when the animal is positioned within the place fields of those cells. The position-based firing properties of hippocampal cells make it very difficult to sample neural
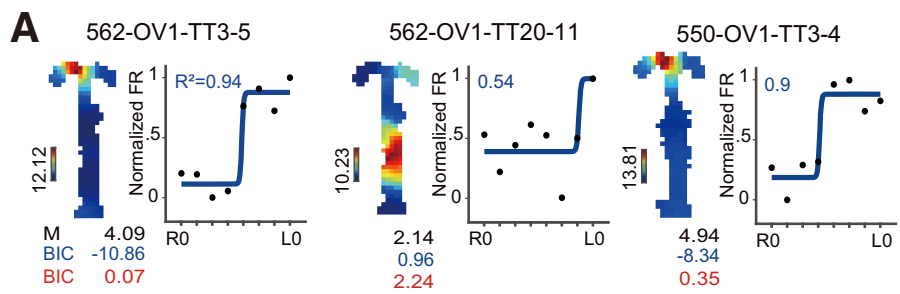

487-OV1-TT7-2

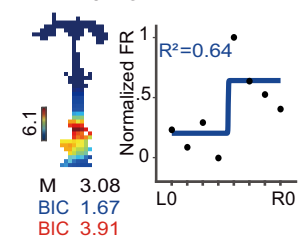

487-OV1-TT8-7

313-OV2-TT4-8
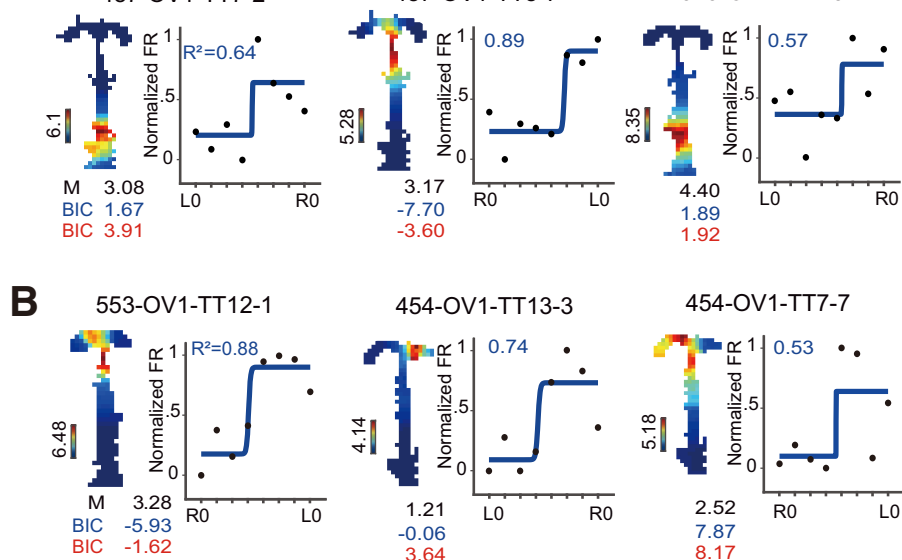

454-OV1-TT7-7

454-OV1-TT23-1

454-OV1-TT22-8
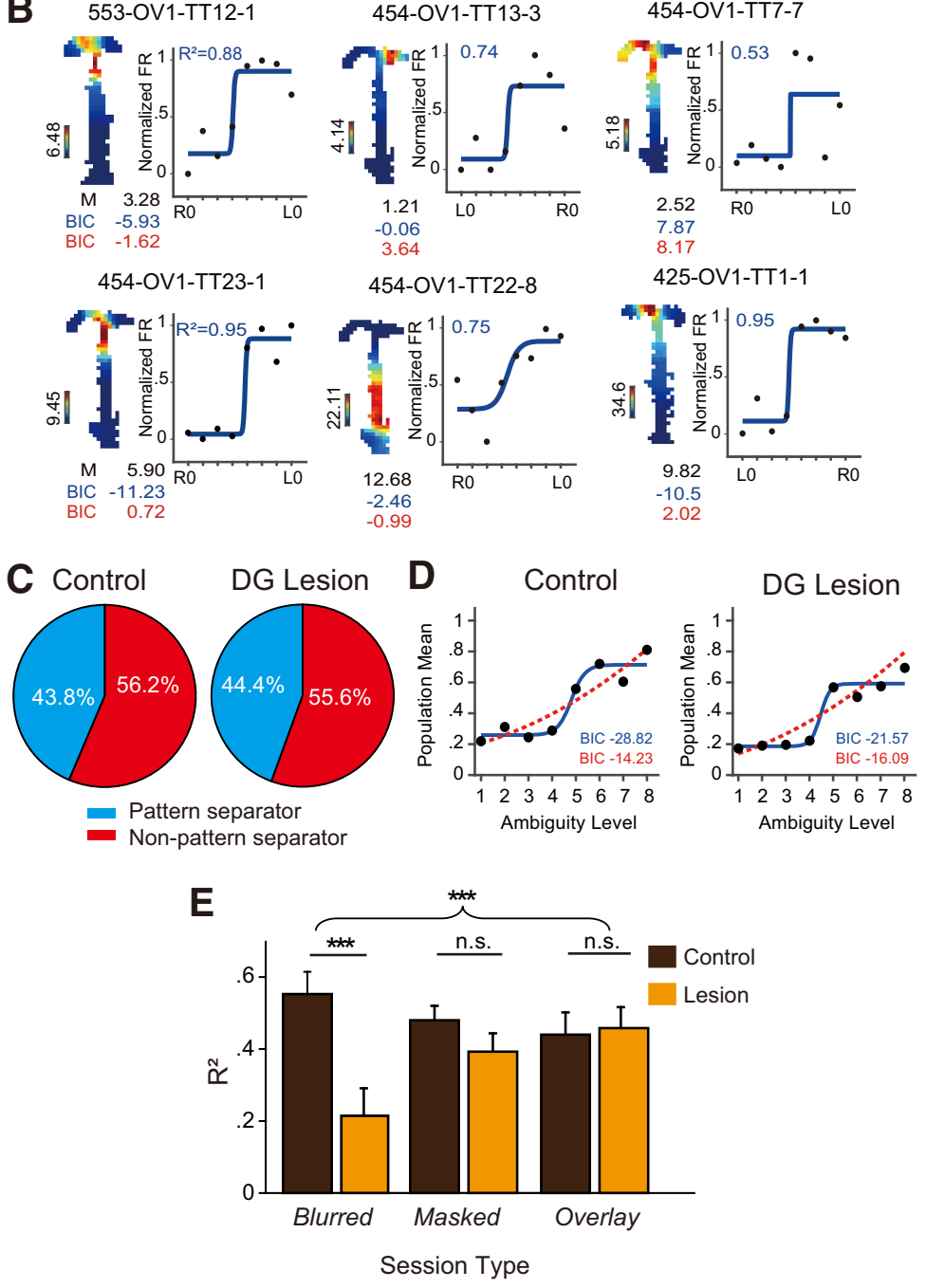

Figure 9. Scene-dependent rate modulation in the CA3 during the retrieval of partially mixed visual scenes. A, Examples of stepwise rate modulation in CA3 units with intact DG, as shown in Figure 6 A. Blue lines indicate well fit sigmodal models. BIC values (blue from the sigmoidal model and red from the quadratic model) used for the categorization are shown below the rate maps. $\boldsymbol{B}$, Examples of categorical rate modulation in CA3 units with DG lesions. C, The proportion of units that were better explained by the sigmoidal model (pattern separator-like cells, blue) and by the quadratic model (non-pattern separator-like units, red) in the control and DG-lesion groups during the Overlay sessions. D, The population scene-tuning curves from control (left) and DGlesion (right) groups in the Overlay sessions. BIC values from the sigmoidal model (blue) and quadratic model (red) are shown below the scene-tuning curve. $\boldsymbol{E}$, The coefficient of determination $\left(R^{2}\right)$ of the sigmoidal model fit from the control and DG-legion groups across the Blurred, Masked, and Overlay sessions. Values are given in mean \pm SE. n.s. not significant, ${ }^{* *} p<0.001$. 
activities from enough cells when a behavioral apparatus requires the rat to perform the task in a fixed area. Second, unlike the results from the human studies (Kirwan and Stark, 2007; Bakker et al., 2008; Lacy et al., 2011; Motley and Kirwan, 2012; Stevenson et al., 2020), prior behavioral studies suggest that object recognition itself may not be hippocampal dependent in rodents (Gilbert and Kesner, 2003; Jo and Lee, 2010). On the other hand, inactivating the perirhinal cortex in the current visual scene task resulted in only minor deficits (Park et al., 2017), compared with the devastating effects of the hippocampal inactivation (data not shown). Therefore, we reasoned that using a task that requires spatial navigation on the basis of visual patterns that can be manipulated similarly as in the study by Ahn and Lee (2017) should provide the best experimental design to physiologically examine pattern separation in the CA3 with or without the DG. The results of the current study, together with our findings in the perirhinal cortex for object recognition (Ahn and Lee, 2017), strongly suggest that the neural networks perform the signature behavior of pattern separation across different regions of the brain when the optimal type of stimulus (e.g., object in the perirhinal cortex, visual scene in the hippocampus) is ambiguously altered from the original pattern.

In the absence of the DG, cells in the CA3 tended to respond gradually to continuously changing ambiguous visual scenes. Inputs from the entorhinal cortex via the perforant path to the CA3 network are likely to scale linearly with the degree of stimulus similarity. The CA1 network also receives direct inputs from the entorhinal cortex, with prior studies showing the relatively linear mapping of environmental ambiguity by CA1 cells (Lee et al., 2004b; Leutgeb et al., 2004; Vazdarjanova and Guzowski, 2004). Our study confirms the nature of these entorhinal inputs to the hippocampus and re-emphasizes the critical roles of the DG in implementing categorical dynamics in information processing using gradually changing inputs from the entorhinal cortex in the hippocampal network. Our findings suggest that the pattern-separating operations of the DG network may be necessary for the acquisition of novel environmental contexts as well as for the retrieval of memory representations when encountering altered environments. Such a pattern-separating computational component may be essential in orchestrating network dynamics to strategically organize behavior for efficient problem solving in a goal-directed task.

\section{References}

Ahn JR, Lee I (2014) Intact CA3 in the hippocampus is only sufficient for contextual behavior based on well-learned and unaltered visual background. Hippocampus 24:1081-1093.

Ahn JR, Lee I (2017) Neural correlates of both perception and memory for objects in the rodent perirhinal cortex. Cereb Cortex 27:3856-3868.

Bakker A, Kirwan CB, Miller M, Stark CEL (2008) Pattern separation in the human hippocampal CA3 and dentate gyrus. Science 319:1640-1642.

Berron D, Schütze H, Maass A, Cardenas-Blanco A, Kuijf HJ, Kumaran D, Düzel E (2016) Strong evidence for pattern separation in human dentate gyrus. J Neurosci 36:7569-7579.

Brown TI, Hasselmo ME, Stern CE (2014) A high-resolution study of hippocampal and medial temporal lobe correlates of spatial context and prospective overlapping route memory. Hippocampus 24:819-839.

Buckmaster PS, Wenzel HJ, Kunkel DD, Schwartzkroin PA (1996) Axon arbors and synaptic connections of hippocampal mossy cells in the rat in vivo. J Comp Neurol 366:271-292.

Danielson NB, Turi GF, Ladow M, Chavlis S, Petrantonakis PC, Poirazi P, Losonczy A (2017) In vivo imaging of dentate gyrus mossy cells in behaving mice. Neuron 93:552-559.e4.
Delcasso S, Huh N, Byeon JS, Lee J, Jung MW, Lee I (2014) Functional relationships between the hippocampus and dorsomedial striatum in learning a visual scene-based memory task in rats. J Neurosci 34:15534-15547.

Eichenbaum H (2000) A cortical-hippocampal system for declarative memory. Nat Rev Neurosci 1:41-50.

Gilbert PE, Kesner RP (2003) Recognition memory for complex visual discriminations is influenced by stimulus interference in rodents with perirhinal cortex damage. Learn Mem 10:525-530.

Gilbert PE, Kesner RP (2006) The role of the dorsal CA3 hippocampal subregion in spatial working memory and pattern separation. Behav Brain Res 169:142-149.

Gilbert PE, Kesner RP, DeCoteau WE (1998) Memory for spatial location: role of the hippocampus in mediating spatial pattern separation. J Neurosci 18:804-810.

Gilbert PE, Kesner RP, Lee I (2001) Dissociating hippocampal subregions: a double dissociation between dentate gyrus and CA1. Hippocampus 11:626-636.

Gonzales RB, DeLeon Galvan CJ, Rangel YM, Claiborne BJ (2001) Distribution of thorny excrescences on CA3 pyramidal neurons in the rat hippocampus. J Comp Neurol 430:357-368.

GoodSmith D, Chen X, Wang C, Kim SH, Song H, Burgalossi A, Christian KM, Knierim JJ (2017) Spatial representations of granule cells and mossy cells of the dentate gyrus. Neuron 93:677-690.e5.

Jinde S, Zsiros V, Jiang Z, Nakao K, Pickel J, Kohno K, Belforte JE, Nakazawa K (2012) Hilar mossy cell degeneration causes transient dentate granule cell hyperexcitability and impaired pattern separation. Neuron 76:11891200.

Jo YS, Lee I (2010) Disconnection of the hippocampal-perirhinal cortical circuits severely disrupts object-place paired associative memory. J Neurosci 30:9850-9858.

Kesner RP, Rolls ET (2015) A computational theory of hippocampal function, and tests of the theory: new developments. Neurosci Biobehav Rev 48:92-147.

Kirwan CB, Stark CE (2007) Overcoming interference: an fMRI investigation of pattern separation in the medial temporal lobe. Learn Mem 14:625633.

Lacy JW, Yassa MA, Stark SM, Muftuler LT, Stark CE (2011) Distinct pattern separation related transfer functions in human CA3/dentate and CA1 revealed using high-resolution $\mathrm{fMRI}$ and variable mnemonic similarity. Learn Mem 18:15-18.

Lee H, Wang C, Deshmukh SS, Knierim JJ (2015) Neural population evidence of functional heterogeneity along the CA3 transverse axis: pattern completion versus pattern separation. Neuron 87:1093-1105.

Lee I, Kesner RP (2003) Differential roles of dorsal hippocampal subregions in spatial working memory with short versus intermediate delay. Behav Neurosci 117:1044-1053.

Lee I, Kesner RP (2004) Encoding versus retrieval of spatial memory: double dissociation between the dentate gyrus and the perforant path inputs into CA3 in the dorsal hippocampus. Hippocampus 14:66-76.

Lee I, Solivan F (2010) Dentate gyrus is necessary for disambiguating similar object-place representations. Learn Mem 17:252-258.

Lee I, Kim J, Lee C (1999) Anatomical characteristics and three-dimensional model of the dog dorsal lateral geniculate body. Anat Rec 256:29-39.

Lee I, Rao G, Knierim JJ (2004a) A double dissociation between hippocampal subfields: differential time course of CA3 and CA1 place cells for processing changed environments. Neuron 42:803-815.

Lee I, Yoganarasimha D, Rao G, Knierim JJ (2004b) Comparison of population coherence of place cells in hippocampal subfields CA1 and CA3. Nature 430:456-459.

Leutgeb JK, Leutgeb S, Moser M-B, Moser EI (2007) Pattern separation in the dentate gyrus and CA3 of the hippocampus. Science 315:961-966.

Leutgeb S, Leutgeb JK, Treves A, Moser MB, Moser EI (2004) Distinct ensemble codes in hippocampal areas CA3 and CA1. Science 305:12951298.

Marr D (1971) Simple memory: a theory for archicortex. Philos Trans R Soc Lond B Biol Sci 262:23-81.

McNaughton BL, Barnes CA, Meltzer J, Sutherland RJ (1989) Hippocampal granule cells are necessary for normal spatial learning but not for spatially-selective pyramidal cell discharge. Exp Brain Res 76:485-496.

Motley SE, Kirwan CB (2012) A parametric investigation of pattern separation processes in the medial temporal lobe. J Neurosci 32:13076-13085. 
Nakashiba T, Cushman JD, Pelkey KA, Renaudineau S, Buhl DL, McHugh TJ, Rodriguez Barrera V, Chittajallu R, Iwamoto KS, McBain CJ, Fanselow MS, Tonegawa S (2012) Young dentate granule cells mediate pattern separation, whereas old granule cells facilitate pattern completion. Cell 149:188-201.

Nakazawa K (2017) Dentate mossy cell and pattern separation. Neuron 93:1236.

Neunuebel JP, Knierim JJ (2014) CA3 retrieves coherent representations from degraded input: direct evidence for CA3 pattern completion and dentate gyrus pattern separation. Neuron 81:416-427.

O'Reilly RC, McClelland JL (1994) Hippocampal conjunctive encoding, storage, and recall: avoiding a trade-off. Hippocampus 4:661-682.

Park EH, Ahn JR, Lee I (2017) Interactions between stimulus and response types are more strongly represented in the entorhinal cortex than in its upstream regions in rats. eLife 6:e32657.

Sakon JJ, Suzuki WA (2019) A neural signature of pattern separation in the monkey hippocampus. Proc Natl Acad Sci U S A 116:9634-9643.

Sasaki T, Piatti VC, Hwaun E, Ahmadi S, Lisman JE, Leutgeb S, Leutgeb JK (2018) Dentate network activity is necessary for spatial working memory by supporting CA3 sharp-wave ripple generation and prospective firing of CA3 neurons. Nat Neurosci 21:258-269.

Schwarz G (1978) Estimating the dimension of a model. Ann Stat 6:461-464.

Senzai Y, Buzsáki G (2017) Physiological properties and behavioral correlates of hippocampal granule cells and mossy cells. Neuron 93:691-704.e5.
Skaggs WE, McNaughton BL, Gothard KM (1993) An information-theoretic approach to deciphering the hippocampal code. Adv Neural Inf Process Syst 6:1030-1037.

Stevenson RF, Reagh ZM, Chun AP, Murray EA, Yassa MA (2020) Pattern separation and source memory engage distinct hippocampal and neocortical regions during retrieval. J Neurosci 40:843-851.

Treves A, Rolls ET (1994) Computational analysis of the role of the hippocampus in memory. Hippocampus 4:374-391.

van Dijk MT, Fenton AA (2018) On how the dentate gyrus contributes to memory discrimination. Neuron 98:832-845.

Vazdarjanova A, Guzowski JF (2004) Differences in hippocampal neuronal population responses to modifications of an environmental context: evidence for distinct, yet complementary, functions of CA3 and CA1 ensembles. J Neurosci 24:6489-6496.

Walsh TJ, Schulz DW, Tilson HA, Schmechel DE (1986) Colchicine-induced granule cell loss in rat hippocampus: selective behavioral and histological alterations. Brain Res 398:23-36.

Wang Z, Bovik AC, Sheikh HR, Simoncelli EP (2004) Image quality assessment: from error visibility to structural similarity. IEEE Trans Image Process 13:600-612.

Xavier GF, Oliveira-Filho FJ, Santos AM (1999) Dentate gyrus-selective colchicine lesion and disruption of performance in spatial tasks: difficulties in "place strategy" because of a lack of flexibility in the use of environmental cues? Hippocampus 9:668-681. 